\title{
Silicon isotope and trace element constraints on the origin of $\sim 3.5$ Ga cherts: Implications for Early Archaean marine environments
}

\author{
S.H.J.M. van den Boorn ${ }^{\mathrm{a}, 1}$, M.J. van Bergen ${ }^{\mathrm{a}, *}$, P.Z. Vroon ${ }^{\mathrm{b}}$, \\ S.T. de Vries ${ }^{\mathrm{a}}$, W. Nijman ${ }^{\mathrm{a}}$ \\ ${ }^{a}$ Department of Earth Sciences, Utrecht University, Budapestlaan 4, 3584 CD, Utrecht, The Netherlands \\ ${ }^{\mathrm{b}}$ Faculty of Earth and Life Sciences, Vrije Universiteit, De Boelelaan 1085, $1081 \mathrm{HV}$, Amsterdam, The Netherlands
}

Received 11 March 2009; accepted in revised form 9 September 2009; available online 19 September 2009

\begin{abstract}
Silicon ( $\mathrm{Si}$ ) isotope variability in Precambrian chert deposits is significant, but proposed explanations for the observed heterogeneity are incomplete in terms of silica provenance and fractionation mechanisms involved. To address these issues we investigated $\mathrm{Si}$ isotope systematics, in conjunction with geochemical and mineralogical data, in three well-characterised and approximately contemporaneous, $\sim 3.5 \mathrm{Ga}$ chert units from the Pilbara greenstone terrane (Western Australia).

We show that $\mathrm{Si}$ isotope variation in these cherts is large $(-2.4 \%$ to $+1.3 \%$ ) and was induced by near-surface processes that were controlled by ambient conditions. Cherts that formed by chemical precipitation of silica show the largest spread in $\delta^{30} \mathrm{Si}(-2.4 \%$ to $+0.6 \%$ on are characterised by positive Eu, La and Y anomalies and overall depletions in lithophile trace elements. Silicon isotope systematics in these orthochemical deposits are explained by (1) mixing between hydrothermal fluids and seawater, and/or (2) fractionation of hydrothermal fluids by subsurface losses of silica due to conductive cooling. Rayleigh-type fractionation of hydrothermal fluids was largely controlled by temperature differences between these fluids and seawater. Lamina-scale $\mathrm{Si}$ isotope heterogeneity within individual chemical chert samples up to $2.2 \%$ is considered to reflect the dynamic nature of hydrothermal activity. Silicified volcanogenic sediments lack diagnostic REE $+\mathrm{Y}$ anomalies, are enriched in lithophile elements, and exhibit a much more restricted range of positive $\delta^{30} \mathrm{Si}(+0.1 \%$ to $+1.1 \%$, which points to seawater as the dominant source of silica.

The proposed model for Si isotope variability in the Early Archaean implies that chemical cherts with the most negative $\delta^{30} \mathrm{Si}$ formed from pristine hydrothermal fluids, whereas silicified or chemical sediments with positive $\delta^{30} \mathrm{Si}$ are closest to pure seawater deposits. Taking the most positive value found in this study $(+1.3 \%)$, and assuming that the Si isotope composition of seawater is governed by input of fractionated hydrothermal fluids, we infer that the temperature of $\sim 3.5 \mathrm{Ga}$ seawater was below $\sim 55^{\circ} \mathrm{C}$.
\end{abstract}

(c) 2009 Elsevier Ltd. All rights reserved.

\section{INTRODUCTION}

\footnotetext{
* Corresponding author. Tel.: +3130 2535036; fax: +3130 2535030.

E-mail addresses: sander.van-den-boorn@shell.com (S.H.J.M. van den Boorn), vbergen@geo.uu.nl (M.J. van Bergen).

${ }^{1}$ Present address: Shell Projects and Technologies, Kessler Park 1, 2288 GS, Rijswijk, The Netherlands.
}

Archaean cherts are the oldest relics of sedimentary deposits and therefore contain unique information on ambient conditions at the surface of the early Earth. However, the provenance of silica - the main constituent of cherts - and the depositional environment remain subject to debate (Buick and Dunlop, 1990; Nijman et al., 1999a; 
Kato and Nakamura, 2003; Knauth and Lowe, 2003; Van Kranendonk, 2006; Van den Boorn et al., 2007). Solving these questions is timely, because cherts host the oldest putative evidence of life on Earth (e.g., Schopf et al., 2002) and have been used to infer ambient ocean temperatures as high as $70{ }^{\circ} \mathrm{C}$ at $3.5 \mathrm{Ga}$ (Knauth and Lowe, 2003; Robert and Chaussidon, 2006).

Recent studies have demonstrated a salient variability of Si isotope ratios in Archaean cherts (André et al., 2006; Robert and Chaussidon, 2006; Van den Boorn et al., 2007; Abraham et al., 2007). Based on a secular increase in $\delta^{30} \mathrm{Si}$ throughout the Precambrian, Robert and Chaussidon (2006) proposed that $\mathrm{Si}$ isotopes record gradually declining oceanic temperatures, corroborating previous inferences from oxygen isotopes (Knauth and Lowe, 2003). In contrast, Van den Boorn et al. (2007) concluded that $\mathrm{Si}$ isotope variability in a suite of 3.5-3.0 Ga cherts reflects different sources of silica and is largely controlled by the mode and environment of deposition.

In order to better constrain the significance of $\mathrm{Si}$ isotope signatures as a probe of Early Archaean surface environments, we studied three well-characterised chert units of the Pilbara greenstone terrane (Western Australia) in detail. The $\sim 3.46$ Ga Marble Bar Chert is believed to represent a setting where cherts formed by orthochemical deposition of silica (C-cherts). In contrast, the $\sim 3.45$ Ga Kitty's Gap Chert is typical for sites where silicification largely proceeded through replacement of volcanogenic sediments (Scherts) (Van den Boorn et al., 2007). The 3.49 Ga North Pole chert-barite unit is intermediate between these two end-member settings, and provides evidence for both mechanisms of chert formation (Van den Boorn et al., 2007).

We show that the selected settings are distinct in terms of $\mathrm{Si}$ isotope and trace element geochemistry (notably $\mathrm{REE}+\mathrm{Y}$ ), and that $\mathrm{km}$ to $\mathrm{mm}$-scale variations in $\mathrm{Si}$ isotope composition are a characteristic feature of Early Archaean chert deposits. We explore the underlying controls of chert formation and $\mathrm{Si}$ isotope heterogeneity, and discuss implications for the temperature of Early Archaean oceans.

\section{GEOLOGICAL BACKGROUND AND SAMPLING STRATEGY}

The Pilbara craton, one of the best-studied Archaean greenstone terranes (Barley, 1993; Van Kranendonk et al., 2002, 2007), spans an area of approximately 530 by $230 \mathrm{~km}$ in Western Australia (Fig. 1). The oldest rocks are exposed in the northern part and are collectively known as the Pilbara Supergroup (3.53-3.17 Ga) which has been subdivided into four groups: (1) the Warrawoona Group (3.53-3.43 Ga), including the oldest preserved felsic rock of the Coonterunah Subgroup; (2) the Kelly Group (no age limits available); (3) the Sulphur Springs Group $(\leqslant 3.27-3.23 \mathrm{Ga})$; and (4) the Soanesville Group (3.23-3.17 Ga) (Van Kranendonk et al., 2007). The cherts studied here belong to the Warrawoona Group wherein regional metamorphic grades generally do not exceed greenschist facies and often only reach prehnitepumpellyite facies (Van Kranendonk et al., 2007).

The Warrawoona Group is a succession of (ultra)mafic and less abundant intermediate to felsic volcanic rocks.
Lenticular bodies of cherts and carbonates are interstratified at different levels. Deposition of extrusive volcanic rocks and intercalated sediments took probably place in a shallow marine environment on a granitoid substrate that is locally preserved below the Coonterunah Subgroup (Buick et al., 1995; Green et al., 2000). Three distinct chert units of the Warrawoona Group were selected for this study, in stratigraphic order: (1) the North Pole chert-barite unit of the Dresser Formation, (2) the Marble Bar Chert of the Towers Formation, and (3) the Kitty's Gap Chert which occurs at the top of the Panorama Formation. Below we briefly discuss the geological context of the samples. Details on sample origins and descriptions are given in Electronic Annex 1 (Table EA-1).

\subsection{Marble Bar Chert}

The Marble Bar Chert marks the boundary between the Coongan and Salgash Subgroups in the Marble Bar Belt. Deposition of the Marble Bar Chert followed a period of pronounced extrusion of felsic volcanics of the Duffer Formation. It is overlain by the Apex Basalt.

The Marble Bar Chert displays a spectacular alternation of predominantly red (jaspilitic), white and black chert. Van Kranendonk (2006) proposed that formation of the Marble Bar Chert started with a phase of stratiform red chert deposition. Fine and regular internal laminations in this jaspilitic variety are indicative of a quiet environment. Red cherts were intruded by white chert as indicated by discordant features and characteristic 'pinch and swell' and replacement structures (Van Kranendonk, 2006). Shortly after deposition of the red and white cherts, the unit was fragmented and brecciated by the intrusion of black chert that occurs as sills and crosscutting veins. Because black cherts do not protrude into the overlying Apex Basalt, post-tilting intrusion (Oliver and Cawood, 2001) is less likely (Van Kranendonk, 2006).

Paucity of detrital mineral phases, dominance of silica and iron and positive Eu anomalies hint at orthochemical precipitation from high-temperature hydrothermal fluids (Kojima et al., 1998; Kato and Nakamura, 2003). The three distinct phases of red, white and black chert deposition probably reflect progressive stages of hydrothermal activity. Some grey chert layers have been associated with the input of detrital sediments (Sugitani, 1992), which would be inconsistent with an inferred MOR setting (Kato and Nakamura, 2003).

Six samples from the Marble Bar Chert were taken at regular intervals from base to top along a $\sim 90 \mathrm{~m}$ road section between the town of Marble Bar and the Comet Gold Mine (Table EA-1). The set includes black (to brown), white (to pink) and red colour varieties. Three of these samples were selected to investigate $\mathrm{Si}$ isotope variability on sub-cm scale (MB-04, MB-03 and MB-10). Sample MB-10 is from a detached boulder of black-white-red banded chert originating from an intermediate level of the section. One sample of silicified basalt was taken from the Apex Basalt stratigraphically above the Marble Bar Chert. 


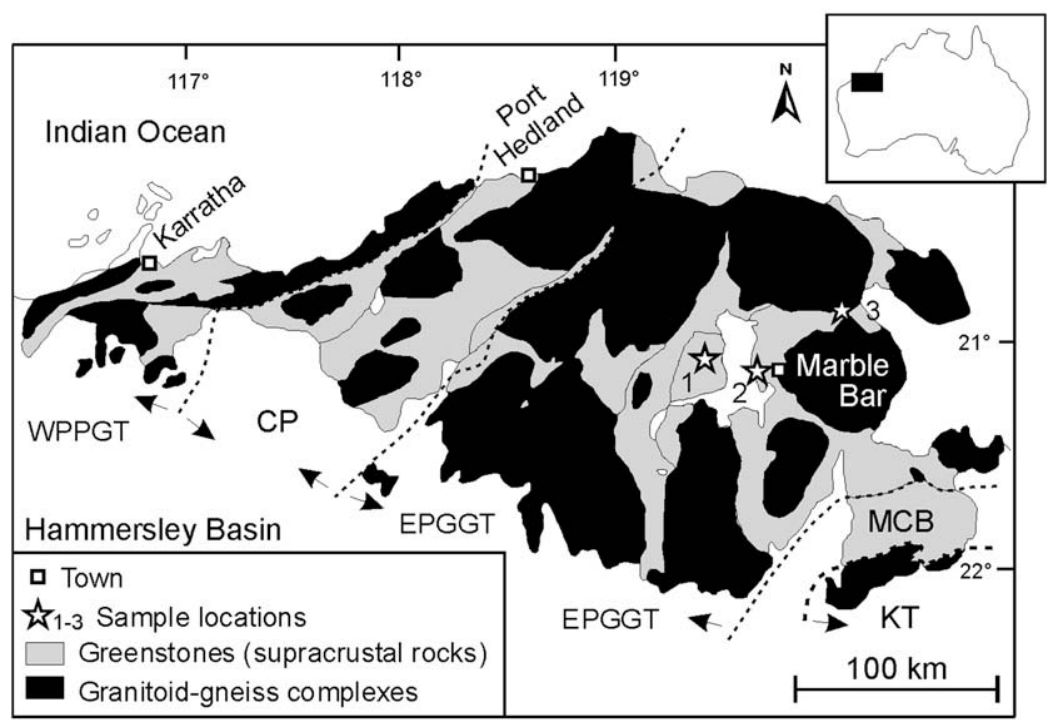

Fig. 1. Simplified geological map (modified after Krapez and Eisenlohr, 1998) of the Pilbara terrane (North-western Australia). Sample locations are indicated with numbers: (1) North Pole; (2) Marble Bar; (3) Kitty's Gap. WPGGT = West Pilbara Granite Greenstone Terrane; $\mathrm{CP}=$ Central Pilbara; EPGGT $=$ East Pilbara Granite Greenstone Terrane; $\mathrm{MCB}=$ Mosquito Creek Basin; KT $=$ Kurrana Terrain $($ after Van Kranendonk et al., 2007).

\subsection{Kitty's Gap Chert}

In the north-eastern part of the Marble Bar Belt an almost complete section trough the Warrawoona Group is exposed in the southern limb of a tightly folded synclinal structure. In this area - also known as the Coppin gap belt (Nijman et al., 1999b) - the Panorama Formation is well exposed. It comprises mainly rhyodacitic to rhyolitic rocks, and is capped by a sequence of silicified sediments, which is known as the Kitty's Gap Chert (De Vries, 2004; De Vries et al., 2006; Orberger et al., 2006; Westall et al., 2006; Rouchon and Orberger, 2008). The Kitty's Gap Chert is overlain by the Euro Basalt.

The cherts occur as an alternation of even-bedded tabular units and fining upward channel fill-shaped bodies composed of silicified black, white and greenish sediments (De Vries, 2004). The cherts are inferred to have a predominantly felsic volcanogenic precursor that underwent syndepositional silicification by interaction with hydrothermal fluids and/or seawater (De Vries, 2004; Orberger et al., 2006; Rouchon and Orberger, 2008). Sedimentary structures such as ripple and flaser-linsen bedding are well preserved in the heterogeneously silicified sequence, and are indicative of a shallow marine depositional environment, probably influenced by tidal activity (De Vries, 2004). Pods of breccia are attributed to fragmentation by explosive hydrothermal activity. Hydrothermal activity in the area is closely associated with normal faulting within the underlying felsic volcanic rock suite. Networks of chert veinlets are observed at depth and develop into metre-wide dikes before they merge into the overlying stratiform chert strata (Nijman et al., 1999b; De Vries, 2004).

Samples from the stratiform Kitty's Gap Cherts were taken at regular intervals within a $\sim 80 \mathrm{~m}$ thick chert unit described in detail by De Vries (2004, her Fig. 4.11a).
Isotopic variability on specimen scale was investigated in groundmass and clasts of brecciated samples (L1-4.0 and L1-73b) and in black and white layers of banded cherts (L1-6A-5 and L1-13.3b). Samples from a dike intersecting the base of the stratiform chert were obtained at different intervals, in upward direction: KG-FC1 (base; $0 \mathrm{~m}$ ), KGFC2 (centre; $\sim 70 \mathrm{~m}$ above base), KG-FC3 ( $\sim 120 \mathrm{~m}$ above base) and KG-FC4 (top; 10 m below stratiform unit). Isotopic heterogeneity between clasts and groundmass was examined in samples KG-FC3 and KG-FC4c. Samples from a margin-centre-margin transect through the $\sim 2 \mathrm{~m}$ wide dike were taken just below the intersection with the stratiform unit (KG-FC4a, KG4-FC4b and KG-FC4c, respectively). A silicified felsic volcanic rock (KG-P1) was collected from the Panorama Formation below the chert, and another silicified extrusive rock (L1-V1) from a volcanic interval within the chert unit (De Vries, 2004).

\subsection{North Pole chert-barite unit}

The North Pole Dome is a granitoid intrusion exposed in a small circular window within the surrounding low-dipping rocks of the overlying Warrawoona Group. Its basal, mainly tholeiitic basalts of the North Star Basalt are overlain by five chert units (North Pole Chert) with intercalated basalts (Nijman et al., 1999a) that collectively form the Dresser Formation (Van Kranendonk et al., 2002). The lowermost, so-called chert-barite unit is particularly well known for its barite beds (Buick and Dunlop, 1990; Nijman et al., 1999a), the preservation of stromatoloidal structures (Walter et al., 1980) and chemical evidence for early biological activity (Ueno et al., 2004, 2006).

The stratiform chert is composed of silicified volcanogenic mud and sandstones, primary silica and silicified gypsum and carbonate. Deposition of these sediments most 
likely occurred in a very shallow marine, evaporitic and/or tidally influenced setting (Buick and Dunlop, 1990; Nijman et al., 1999a). Field observations contradict claims for water depths exceeding $1600 \mathrm{~m}$ based on mineral assemblages in altered greenstones (Kitajima et al., 2001).

Pervasive hydrothermal activity is believed to have been responsible for silicification and baritisation of the preexisting sediments, and resulted in orthochemical precipitation of silica and barite. Networks of chert-barite dikes that crosscut the underlying basaltic succession at high angle and terminate in the chert-barite unit are associated with growth faulting and believed to represent ancient conduits of rising hydrothermal fluids (Nijman et al., 1999a; Van Kranendonk, 2006). The non-protruding nature of these dikes as well as alteration patterns in the surrounding volcanic successions corroborate claims for syndepositional silicification (and baritization) (Van Kranendonk and Pirajno, 2004). Ueno et al. (2004) divided the chert dikes into pre-metasomatic black chert with a sulphidic mineral assemblage and post-metasomatic grey chert that is essentially free of sulphides. The predominance of silica (and barite) and the paucity of base metals suggest a rather low-temperature nature of the hydrothermal venting system ( 100-200 ${ }^{\circ} \mathrm{C}$; Buick and Dunlop, 1990; Nijman et al., 1999a; Ueno et al., 2004). Fluid-inclusion data from quartz crystals in the cherts illustrate that mixing of hydrothermal fluids and seawater was intrinsic to the formation of the North Pole Chert (Foriel et al., 2004; Nishizawa et al., 2007).

Two stratiform chert samples were taken from the North Pole chert-barite unit. Sample NP-CU5 belongs to the Coarsening Upward sandstone $(\mathrm{Cu}-\mathrm{S})$ facies in the locality illustrated in Fig. 11 of Nijman et al. (1999a). Both the bulk sample (NP-CU5B) and the translucent groundmass (NP-CU5), in which the sandstone grains occur, were analysed for $\mathrm{Si}$ isotopic composition. The second sample (VM180T) is characterised by a rhythmic alternation of white-red-pink chert, and belongs to the Even Laminated Chert (ELC) facies in the basal part of $\log 2$ in Fig. 8 of Nijman et al. (1999a).

A number of black and grey samples of dike chert were collected from various exposures in the North Pole area. Samples NP-FC3, NP-FC8 and NP-FC9 come from a dike that intersects the base of the stratiform chert at the locality where sample NP-CU5 was taken. Samples NP-FC11b and NP-FC12 were taken from two dikes at the entrance of the Dresser Mine. Characteristic veins of grey chert, as described by Ueno et al. (2004), occur in the black groundmass of sample NP-FC12c. Sample NP-FC14 was taken from another dike exposure in the vicinity of the Dresser Mine.

\section{ANALYTICAL METHODS}

\subsection{Silicon isotopes}

A micro-drilling technique (using 1.0 and $1.8 \mathrm{~mm}$ diamond braces) was employed to obtain sample material from polished rock slabs. Silicon isotope ratios were measured in duplicate using two separate drill sites. Details of the
$\mathrm{NaOH}$ sample digestion procedure and MC-ICP MS setup for $\mathrm{Si}$ isotope analysis are outlined in Van den Boorn et al. (2006). Sample powder was digested in sodium hydroxide monohydrate $\left(\mathrm{NaOH} \cdot \mathrm{H}_{2} \mathrm{O}\right)$ at $200^{\circ} \mathrm{C}$ in Parr bombs. Digested sample was dissolved in milli-Q water and purified using AG50-X8 cation exchange resin. In case of incomplete dissolution, residue and supernatant were separated by centrifuging, and the residue was treated with $7 \mathrm{~N} \mathrm{HNO}_{3}$ and, if necessary, again with $\mathrm{NaOH} \cdot \mathrm{H}_{2} \mathrm{O}$.

Silicon isotope ratios were determined with a ThermoFinnigan Neptune MC-ICP MS (at the Vrije University, Amsterdam) in medium resolution mode (Resolving Power $=2500$ ). Measurements were performed with $2-$ $5 \mathrm{ppm} \mathrm{SiO}_{2}$ in $1 \% \mathrm{HNO}_{3}$ solutions, and blanks were always $<1 \%$ of the intensity of samples or standards (Van den Boorn et al., 2006). Silicon isotope data are reported as the permil deviation from the NIST RM-8546 (formerly known as NBS-28) standard, using a standard-sample bracketing technique. The Diatomite standard was included in every sequence to check for accuracy and precision, and yielded $+1.37 \pm 0.13$ ( $1 \mathrm{SD}, n=109$ ) (cf., Reynolds et al., 2007). All our data plot near a mass-dependent fractionation line with a slope of 1.95 , which is between the value for equilibrium (1.93) and kinetic fractionation (1.96), testifying that all polyatomic interferences are resolved.

\subsection{Major and trace elements}

About 5-10 mg of sample material was drilled from polished rock slabs and split into fractions for major and trace element analysis. In addition, whole-rock analyses were obtained for samples NP-CU5B, L1-V1, KG-P1 and APB2. These samples were crushed in a steel jaw crusher and grinded in a steel shatterbox, which precluded the analysis of trace metals. Micro-drilled samples were digested overnight in a mixture of $\sim 0.5 \mathrm{ml}$ concentrated Teflon-distilled (TD) $\mathrm{HNO}_{3}$ and $\sim 0.2 \mathrm{ml} \mathrm{40 \%}$ TD HF in closed Savillex beakers at $130{ }^{\circ} \mathrm{C}$. Whole-rock samples were digested under similar conditions but with $\sim 100 \mathrm{mg}$ of sample material in $4 \mathrm{ml}$ concentrated TD $\mathrm{HNO}_{3}$ and $1 \mathrm{ml}$ TD $40 \% \mathrm{HF}$. Digested samples were evaporated to dryness, taken up in $\sim 0.1 \mathrm{ml}$ (micro-drilled) or $\sim 1 \mathrm{ml}$ (whole-rock) concentrated TD $\mathrm{HNO}_{3}$ and dried again. This step was repeated once. Micro-drilled samples were then dissolved in $3-5 \mathrm{ml}$ of $1 \%$ TD $\mathrm{HNO}_{3}$. This digestion-dissolution procedure ensures that dilution factors are $<1000$, which facilitates major and trace element analysis in relatively pure chert samples. Whole-rock samples were dissolved in $3 \mathrm{~N} \mathrm{TD} \mathrm{HNO}_{3}$ and then diluted to yield a final $1 \% \mathrm{HNO}_{3}$ solution.

Major-element compositions were determined by ICPAES using a Spectro CIROS CCD instrument equipped with radial plasma source (Utrecht University.) Since the use of HF in the digestion step precludes the analysis of silica, its concentrations were determined in the purified $\mathrm{NaOH}$ solutions for isotope analysis (Van den Boorn et al., 2006). Reported silica concentrations are averages of the duplicate samples (see above). Typical precision of ICP-AES analysis was 2\% (1 SD). For most chert samples the sum of major oxides was $>96 \%$. Occasional lower totals might be caused by the heterogeneous distribution of acces- 
sory minerals. Therefore, all major element data were normalised to a total sum of $100 \%$.

Trace elements were measured by ICP-MS with an Agilent HP4500plus (Vrije University) using a method comparable to the one described by Eggins et al. (1997). BHVO-2 was used as calibration standard and BCR-2 as monitor for accuracy and precision. Typical precision for ICP-MS analysis was $\sim 3-6 \%$ ( $1 \mathrm{SD}$ ) based on 8 different runs of BCR-2.

\subsection{Mineral analysis}

Selected samples were cut into $3 \times 4 \mathrm{~cm}$ slabs using a diamond saw. Thin sections were prepared from the slabs that were subsequently used for micro-drilling (see above). The reported mineralogical and petrological data were obtained by optical microscopy and by EDS analysis using a JEOL JXA8600 electron microprobe at Utrecht University. Determinations of $\mathrm{Si}$, Ti, $\mathrm{Al}, \mathrm{Fe}, \mathrm{Mg}, \mathrm{Mn}, \mathrm{Ca}, \mathrm{Na}, \mathrm{K}, \mathrm{P}$ and $\mathrm{S}$ were performed at $15 \mathrm{kV}$ and $20 \mathrm{nA}$, using a spot size of $1 \mu \mathrm{m}$.

\section{RESULTS}

Mineral assemblages are presented in Electronic Annex 1 (Table EA-1), and major and trace-element data in Electronic Annex 2 (Table EA-2). Most of the samples studied are chiefly composed of $\mathrm{SiO}_{2}(19-100 \%)$ with minor amounts of $\mathrm{Al}_{2} \mathrm{O}_{3}, \mathrm{~K}_{2} \mathrm{O}$ and $\mathrm{TiO}_{2}$ or $\mathrm{FeO}_{t}$. Concentrations of other 'major' elements are negligible or below detection limits of our ICP-AES technique. Fig. 2 shows normalised REE patterns where yttrium is included, given its similarity to Ho (cf., Bau, 1996; Bolhar et al., 2004). Queensland Mud (MUQ; Kamber et al., 2005) is used for normalisation (Bolhar et al., 2005). In the following description the subscript $\mathrm{SN}$ refers to shale (i.e., MUQ) normalised. Normalisation against Post-Archaean Australian Shale (PAAS) might yield slightly different REE $+Y$ patterns but would not affect overall trends and inferences (see also Van Kranendonk et al., 2003; Bolhar et al., 2004). Distinct La, Ce, Eu and/or $\mathrm{Y}$ anomalies, visible in many of the REE+Y patterns, are quantified following Bolhar et al. (2004, after Bau and Dulski, 1996).

Silicon isotope data for 53 (sub)samples from 29 handspecimen are graphically shown in Fig. 3 and listed in Table 1. The data were obtained in duplicate from at least two micro-drilling spots, and are reported separately. For comparison, the $\mathrm{Si}$ isotope range of modern reservoirs and previous results from $>3 \mathrm{Ga}$ rocks are shown in Fig. 4. Precision of the Si measurements was better than $\sim 0.15 \%$ ( $1 \mathrm{SD}$ ), based on multiple analyses of individual sample solutions, and is consistent with our long-term precision of $\sim 0.15 \%$ on the Diatomite standard (Van den Boorn et al., 2006, 2007). Duplicate measurements of separate micro-drilled samples were generally within $0.15 \%$ ( $1 \mathrm{SD}$ ) as well. Occasional differences exceeding this value point to isotopic heterogeneity within individual laminae and clasts. Preliminary laserablation data, revealing isotopic inhomogeneity up to $0.5 \%$ within single laminae in sample VM180T (Vroon et al., 2007), corroborate this. Mean $\delta^{30} \mathrm{Si}$ values of duplicate analyses as reported in Table 1 will be used in the following discussion.

\subsection{Marble Bar Chert}

The Marble Bar Chert samples are characterised by the presence of $\mathrm{Fe}$-(hydr)oxides (mainly goethite and hematite) and minor sulphides in a dominantly microcrystalline groundmass (cf., Sugitani, 1992; Kojima et al., 1998; Kato and Nakamura, 2003). Consequently, their composition is governed by $\mathrm{SiO}_{2}$ and $\mathrm{FeO}_{t}$, which are negatively correlated. A total $\mathrm{FeO}_{t}$ range of $0.05-81 \%$ can be inferred if samples are included that were only analysed for $\mathrm{SiO}_{2}$ (assuming $\mathrm{SiO}_{2}+\mathrm{FeO}_{t}=100 \%$ ).

White cherts of almost pure silica consist of microcrystalline quartz and rare Fe-(hydr)oxides. In contrast, red layers contain significant amounts of Fe-(hydr)oxides (mainly hematite), while in some black-brown layers Fe-(hydr)oxides dominate over quartz. Variable iron contents in red and black-brown layers reach $30 \%$ and $80 \% \mathrm{FeO}_{t}$, respectively. The Fe-rich layers tend to be enriched in $\mathrm{Ni}$ (33$160 \mathrm{ppm}), \mathrm{Zn}$ (20-54 ppm), Cu (13-310 ppm), Co (5$6 \mathrm{ppm})$, $\mathrm{Ba}(3-26 \mathrm{ppm})$ and $\mathrm{Pb}(3-5 \mathrm{ppm})$. An exception is sample $\mathrm{MB}-01$ with low $\mathrm{FeO}_{t}(0.05 \%)$ but high $\mathrm{Ni}$ (110 ppm) and $\mathrm{Ba}(84 \mathrm{ppm})$ contents.

Sericite, Ti-oxide and zircon are virtually absent, as is reflected by low concentrations of $\mathrm{Al}_{2} \mathrm{O}_{3}(0.01-0.17 \%), \mathrm{K}_{2} \mathrm{O}$ $(0-0.02 \%)$ and lithophile trace elements: Th (1-26 ppb), Rb (13-64 ppb), Hf (1-14 ppb), Ga (18-400 ppb), Nb (2$33 \mathrm{ppb})$ and $\mathrm{Zr}(48-640 \mathrm{ppb})$. The low concentrations of $\mathrm{Th}, \mathrm{Zr}$ and $\mathrm{Hf}$ are in the range of those reported for BIF's from the Isua belt that have been interpreted as chemical precipitates with negligible crustal contamination (Bolhar et al., 2004).

Normalised REE $+Y$ patterns of the Marble Bar Chert samples show distinct shapes with noticeable convex LREE depletions $\left(0.2<\operatorname{Pr}_{\mathrm{SN}} / \mathrm{Yb}_{\mathrm{SN}}<0.7\right.$, mean $\left.=0.3\right)$ and strong $\mathrm{Eu}$ anomalies $\left(2.2<\mathrm{Eu} / \mathrm{Eu}_{\mathrm{SN}}^{*}<3.7\right.$, mean $\left.=2.7\right)$. Lanthanum $\left(0.9<\mathrm{La} / \mathrm{La}_{\mathrm{SN}}^{*}<2.1\right.$, mean $\left.=1.5\right)$ and $\mathrm{Y} / \mathrm{Ho}$ $(31<\mathrm{Y} / \mathrm{Ho}<55$; mean $=44)$ anomalies are more variable but mostly positive (Fig. 2a). There are no obvious differences between white, red and black layers, although HREE trends tend to be flatter in white layers.

The $\mathrm{Si}$ isotope compositions (expressed as $\delta^{30} \mathrm{Si}$ ) of the Marble Bar Chert samples span a range between $-1.4 \%$ and $+0.6 \%$. Variations up to $1.3 \%$ occur on lamina scale (Fig. 3 and Table 1). Red layers cover the entire range within the samples $(-1.4 \%$ to $+0.6 \%$, mean $-0.2 \%)$, whereas white $(-0.6 \%$ to $0.0 \%$, mean $=-0.3 \%$ oo and black-brown $(+0.2 \%$ to $+0.6 \%$, mean $=+0.4 \%)$ varieties show more limited spread (Fig. 3). Occasional poor reproducibility between individual micro-drilled samples (e.g., MB-03-B) probably reflects intra-lamina heterogeneity.

\subsection{Kitty's Gap Chert}

The volcaniclastic sedimentary nature of the protolith of the Kitty's Gap Chert is reflected in its mineralogy and geochemistry. The stratiform chert samples of this unit are characterised by impurities of dominantly sericite, and accordingly have higher $\mathrm{Al}_{2} \mathrm{O}_{3}(0.7-9.3 \%)$ and $\mathrm{K}_{2} \mathrm{O}(0.2-$ $2.6 \%$ ) contents, which correlate negatively with $\mathrm{SiO}_{2}$. Elevated $\mathrm{TiO}_{2}(0.01-0.5 \%), \mathrm{Zr}(8-88 \mathrm{ppm})$ and $\sum \mathrm{REE}+\mathrm{Y}$ 
(2-87 ppm) contents signal a close association of Ti-oxide, zircon and, less frequently, monazite and apatite with sericite. Other lithophile elements pointing to crustal precursor material are Th $(0.06-4 \mathrm{ppm}), \mathrm{Rb}(4-41 \mathrm{ppm})$, Hf $(0.2-$ $2 \mathrm{ppm})$, Ga (0.6-10 ppm) and $\mathrm{Nb}(0.04-7 \mathrm{ppm})$, most of
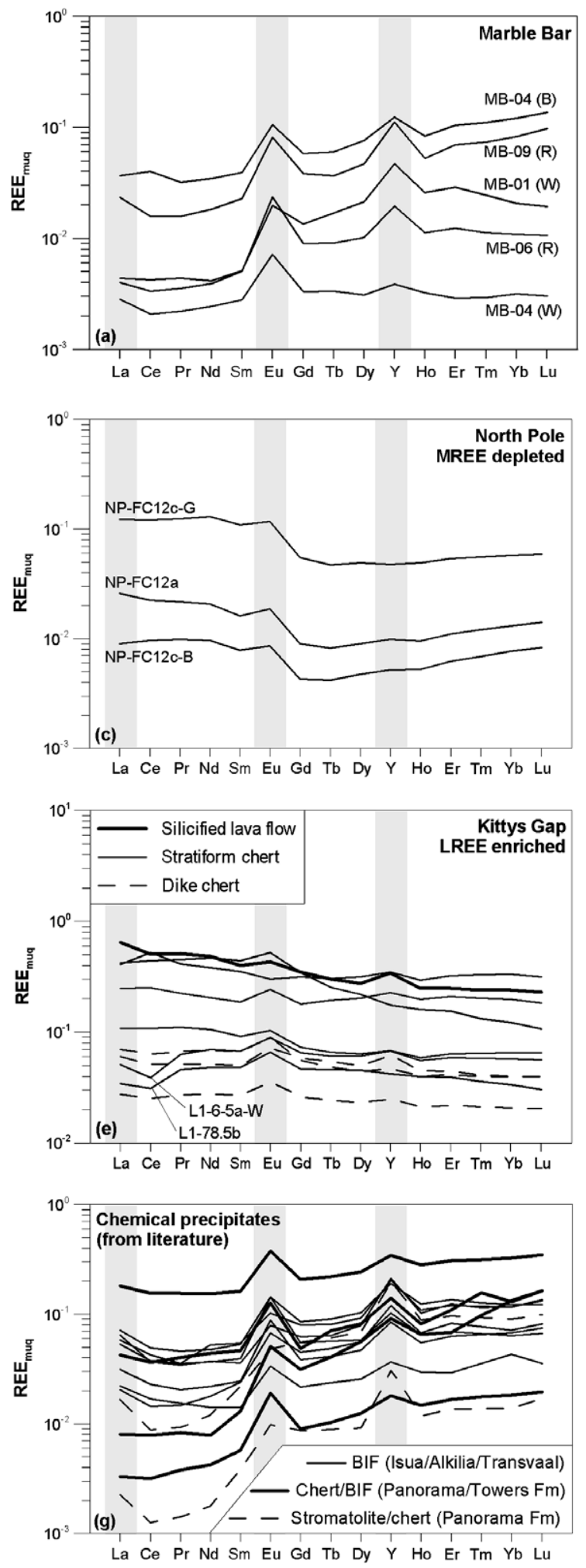

which tend to correlate positively with $\mathrm{Al}_{2} \mathrm{O}_{3}$. Sericite is anhedral to euhedral, and sometimes appear to be pseudomorphs of lath-shaped precursor minerals, presumably feldspar. Occasionally, clusters of Ti-oxides form contours of ghost phases. Minor amounts of detrital quartz, sul-
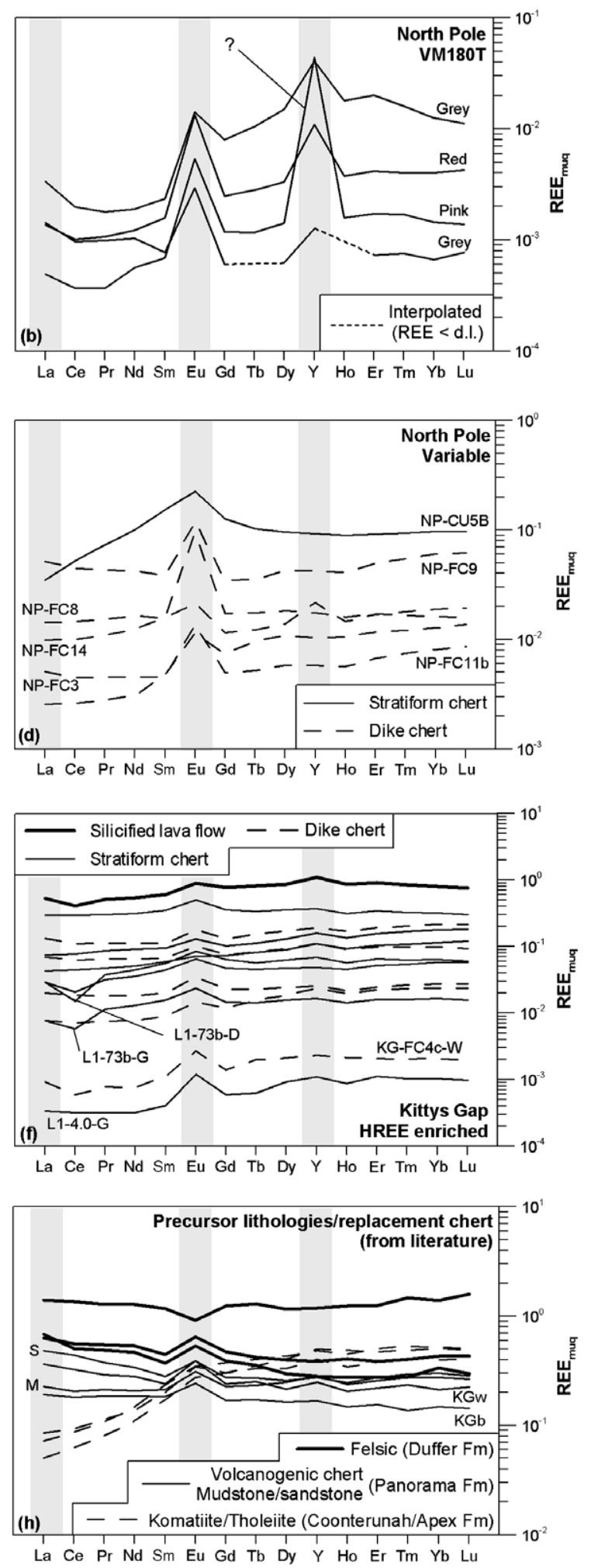
phide/(hydr)oxide and barite are dispersed throughout the various chert layers (cf., Orberger et al., 2006; Westall et al., 2006). Black chert laminae contain appreciable amounts of carbonaceous material, which has been attributed to microbial activity (Westall et al., 2006). Mineral assemblages of characteristic white and black chert bands in the lower part of chert unit are almost identical, but white layers are generally coarser grained and more enriched in accessory minerals. Towards the top of the sequence, greenish cherts dominate (L1-73, L1-78b and KG-C3) and show enrichments in Cr (240-520 ppm) and/ or Co $(0.2-6.5 \mathrm{ppm})$ relative to lower stratigraphic horizons, in line with the presence of ( $\mathrm{Fe}, \mathrm{Cr}$ )-oxides.

The mineralogy of dike chert samples is similar to that of the stratiform cherts but the abundance of accessory minerals and $\mathrm{Al}_{2} \mathrm{O}_{3}(0.2-2.0 \%)$ and $\mathrm{K}_{2} \mathrm{O}(0.06-0.5 \%)$ contents are generally lower. Accordingly, they show more moderate enrichments for $\mathrm{Th}(0.09-1.2 \mathrm{ppm}), \mathrm{Rb}(2.0-$ $12 \mathrm{ppm})$, Hf (0.1-1.4 ppm), Ga (0.6-5.6 ppm), Nb (0.1$2.5 \mathrm{ppm})$ and $\mathrm{Zr}(4.7-60 \mathrm{ppm})$.

The REE + Y patterns of the Kitty's Gap Chert samples can broadly be subdivided into two groups. One shows a relative enrichment in LREE $\left(1.1<\mathrm{Pr}_{\mathrm{SN}} / \mathrm{Yb}_{\mathrm{SN}}<3.7\right.$, mean =1.6; Fig. 2e) while the other exhibits HREE enrichments over LREE and MREE $\left(0.3<\mathrm{Pr}_{\mathrm{SN}} / \mathrm{Yb}_{\mathrm{SN}}<0.9\right.$, mean $=0.6$; Fig. 2f). Cherts from both groups often show subtly positive $\mathrm{Eu}$ anomalies $\left(0.9>\mathrm{Eu} / \mathrm{Eu}_{\mathrm{SN}}^{*}>1.6\right.$, mean $=1.4)$. Variations in $\mathrm{Y} / \mathrm{Ho}$ ratios are generally small $(27<\mathrm{Y} / \mathrm{Ho}<32$, mean $=31)$ and are close to the chondritic value of 26 , except for dike sample KG-FC2 (Y/ $\mathrm{Ho}=36$ ). Anomalies of $\mathrm{Eu}$ and $\mathrm{Y} / \mathrm{Ho}$ are considerably smaller than in the Marble Bar Chert samples. Pronounced negative $\mathrm{Ce}$ anomalies $\left(0.5<\mathrm{Ce} / \mathrm{Ce}_{\mathrm{SN}}^{*}<0.7\right)$ are a remarkable feature in some stratiform chert samples from Kitty's Gap. None of the Kitty's Gap Chert samples shows conspicuous positive $\mathrm{La}$ anomalies $\left(0.8<\mathrm{La} / \mathrm{La}_{\mathrm{SN}}^{*}<1.2\right.$, mean $=1.0$ ).

Sericite and Ti-oxide minerals are lacking in two white to grey/translucent patches in Kitty's Gap Chert samples L1-4.0 and KG-FC4c (i.e., L1-4.0-G and KG-FC4c-W). These patches are composed of almost pure microcrystalline quartz with rare opaque phases (sulphide or (hydr)oxide) and/or barite, but they occur in specimen that are otherwise characterised by sericite, Ti-oxide and zircon.
Both patches have low REE $+\mathrm{Y}$ contents and relatively prominent Eu anomalies (1.9 and 2.6, respectively), whereas $\mathrm{Y} / \mathrm{Ho}$ ratios fall in the range of other Kitty's Gap Chert samples (Fig. 2f).

Kitty's Gap Chert samples show a much more limited spread in $\delta^{30} \mathrm{Si}(-0.2 \%$ to $+1.1 \%)$ compared to Marble Bar and North Pole, and lack the pronounced negative $\delta^{30} \mathrm{Si}$ values. No systematic offset in $\delta^{30} \mathrm{Si}$ between dike and stratiform chert samples is observed, both groups showing roughly the same range (Fig. 3). Isotopic variations between individual laminae and between clasts and groundmass are smaller than in cherts from the other two sites. Subtle isotopic heterogeneity exists between slightly ${ }^{30} \mathrm{Si}$-enriched black layers and ${ }^{30} \mathrm{Si}$-depleted white layers in black-white banded chert samples (L1-6A-5 and L113.3b). The two patches of almost pure silica (L1-4.0-G and KG-FC4c-W) are the only two Kitty's Gap Chert samples with slightly negative $\delta^{30} \mathrm{Si}$.

\subsection{North Pole Chert}

The silicified-sandstone sample (NP-CU5) of the North Pole Chert consists of relatively pure microcrystalline quartz with $\mathrm{Fe}-\mathrm{Mg}$ silicates outlining the coarse sandstone grains. The grains often have a core of microcrystalline quartz. Occasionally, carbonate minerals were observed 'floating' in the microcrystalline groundmass. Only bulk trace-element data (excluding trace metals, see Section 3.2) are available for this sample, of which LILE abundances tend to be lower than in the Kitty's Gap Chert samples. The REE $+Y$ pattern shows a distinct enrichment of HREE and MREE over LREE, a smooth increase from La to $\mathrm{Sm}$ and a small positive Eu anomaly $\left(\mathrm{Eu} / \mathrm{Eu}_{\mathrm{SN}}^{*}=1.7\right)$ (Fig. 2d). The $\delta^{30} \mathrm{Si}$ of both translucent groundmass and bulk sample is positive, but there is an offset of $\sim 0.5 \%$ (Fig. 3).

The geochemical and mineralogical composition of white-pink-red banded sample VM180T closely resembles that of Marble Bar Chert samples. White to translucent grey laminae in stratiform chert sample VM180T are composed of chalcedonic quartz which grades into microcrystalline quartz towards the margins of the laminae. Impurities of Fe-(hydr)oxides are rare. Chalcedonic quartz is characterised by spherulitic blades with undulose extinc-

Fig. 2. MUQ-normalised REE $+\mathrm{Y}$ plots of (a) Marble Bar Chert with bowl-shaped LREE depletions, distinct positive Eu, Y/Ho and (frequent) La anomalies; (b) four different laminae in North Pole sample VM180T with patterns similar to those of Marble Bar samples; extreme Y enrichment (indicated by question mark) remains unexplained; (c) three samples from a single North Pole dike showing MREE depletions and small positive Eu anomalies; (d) North Pole dike and stratiform samples; patterns of FC3, FC8 and FC9 are relatively flat and show positive Eu anomalies; patterns of FC11b and FC14 are roughly similar to Marble Bar samples and VM180T, but FC11b lacks characteristic Y/Ho and La anomaly and FC14 bowl-shaped LREE depletion; stratiform sample NP-CU5B displays smoothly increasing LREE contents, a modest Eu anomaly and flat HREE; (e) Kitty's Gap Cherts with LREE enrichments similar to silicified extrusive-rock sample L1-V1 (bold line) and small variable Eu anomalies; note pronounced Ce anomaly in L1-6A-5-W and L1-78.5b; (f) Kitty's Gap Cherts with slight HREE enrichments similar to silicified lava flow KG-P1 (bold line) and variable small Eu anomalies; note the Ce anomaly in subdomains of sample L1-73b; (g) (averaged) REE patterns of chemical precipitates compiled from the literature (Bau and Dulski, 1996; Kato and Nakamura, 2003; Van Kranendonk et al., 2003; Bolhar et al., 2004, 2005; Orberger et al., 2006; Friend et al., 2008); (h) Typical Archaean felsic and mafic magmatic rocks (Green et al., 2000; Kato and Nakamura, 2003; Van der Meer, 2002), replacement cherts from Kitty's Gap (Orberger et al., 2006; KGw = white layer; $\mathrm{KGb}=$ black layer), and Panorama mudstone $(\mathrm{M})$ and sandstone (S) from Kato and Nakamura (2003). Note that $y$-axes in all plots span three orders of magnitude except in (f). Grey bands indicate anomalous REE+Y anomalies. MUQ values from Kamber et al. (2005). 


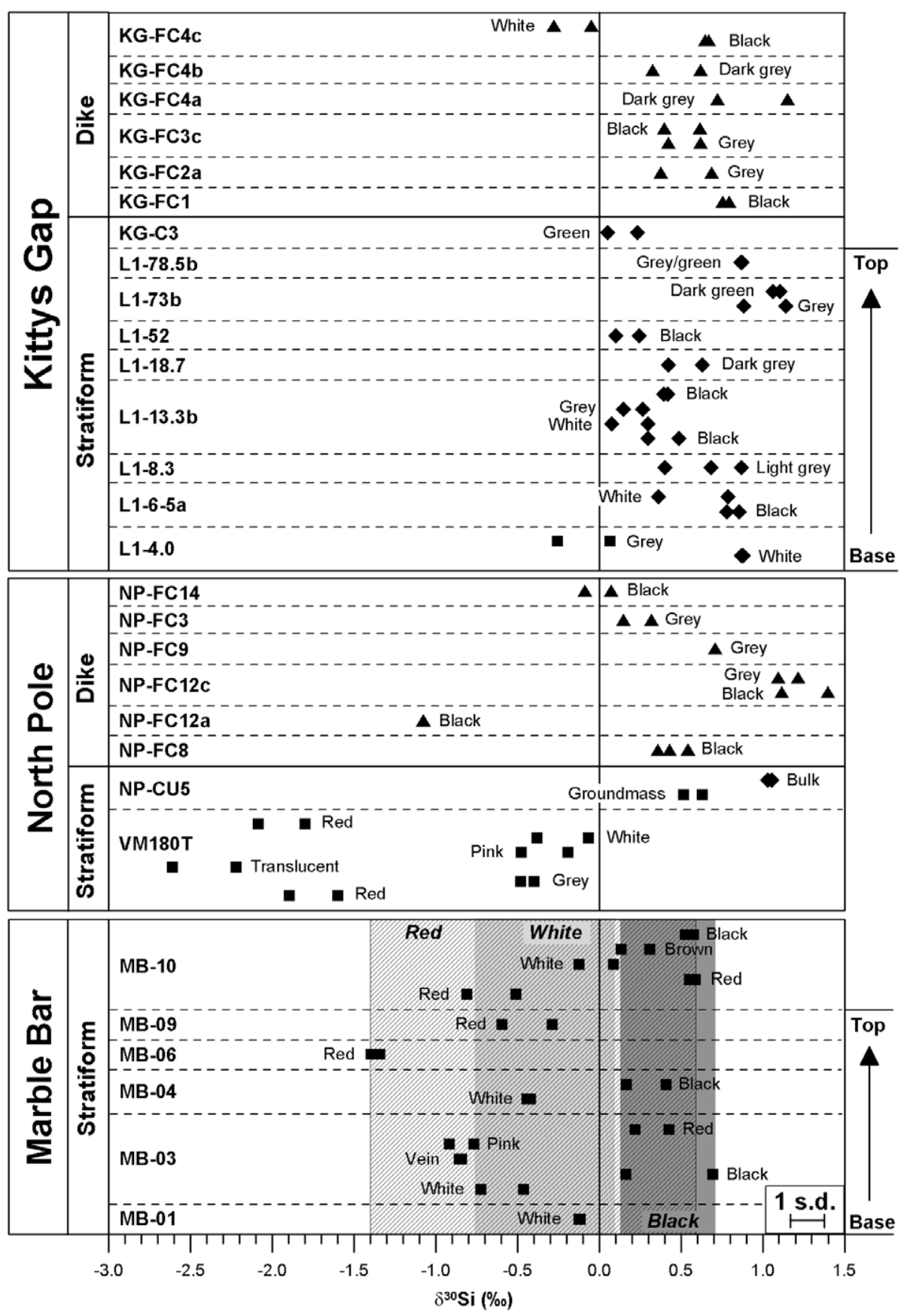

Fig. 3. Silicon isotope variation in Early Archaean cherts from Marble Bar, North Pole and Kitty's Gap. Samples are indicated by codes and are separated by dotted lines. Results for distinct domains (laminae/clasts) are labelled with their colour. Symbols indicate data points for individual micro-drilled spots; $\delta^{30} \mathrm{Si}$ values were obtained for at least two spots in each sample or domain. Squares: stratiform C-cherts; diamonds: stratiform S-cherts; triangles: dike cherts. Long-term $1 \mathrm{SD}$ reproducibility is shown in the lower right corner. Light and dark grey shades and hatched area indicate approximate ranges of white(/grey/pink), black(/brown) and red stratiform C-cherts in the Marble Bar Chert, respectively.

tion. Pink and red layers are composed of microcrystalline quartz and contain larger amounts of Fe-(hydr)oxides. Concentrations of major oxides other than $\mathrm{SiO}_{2}$ and $\mathrm{FeO}_{t}$, notably $\mathrm{Al}_{2} \mathrm{O}_{3}$ and $\mathrm{K}_{2} \mathrm{O}$, are below detection limit. Lithophile trace element contents are low as well: Th (1-2 ppb), $\mathrm{Rb}$ (19-37 ppb), Hf (1-76 ppb), Ga (30-400 ppb), Nb (3$20 \mathrm{ppb})$ and $\mathrm{Zr}(150-4300 \mathrm{ppb})$. Other trace metals such as $\mathrm{Ni}(120-730 \mathrm{ppm})$ and $\mathrm{Ba}(70-200 \mathrm{ppm})$ show variable concentration levels. The REE $+\mathrm{Y}$ patterns of individual laminae in sample VM180T (Fig. 2b) resemble those of the Marble Bar Chert samples in having positive $\mathrm{Eu}$ $\left(2.8<\mathrm{Eu} / \mathrm{Eu}_{\mathrm{SN}}^{*}<6.7\right.$, mean $\left.=5.2\right), \mathrm{Y} / \mathrm{Ho}(59<\mathrm{Y} / \mathrm{Ho}<77$, mean $=68)$ and $\mathrm{La}\left(1.6<\mathrm{La} / \mathrm{La}_{\mathrm{SN}}^{*}<2.1, \quad\right.$ mean $\left.=1.9\right)$ anomalies in all layers. They also display the characteristic 
Table 1

Silicon isotope data for Early Archaean cherts and volcanics from the North Pole, Marble Bar and Kitty's Gap. Both $\delta^{29} \mathrm{Si}$ and $\delta^{30} \mathrm{Si}$ are given for individual micro-drilled spots. If applicable, $1 \mathrm{SD}$ on multiple analyses of individual sample solutions $(n)$ is shown. $\delta^{30} \mathrm{Si}_{\text {mean }}$ is the mean for multiple drill spots.

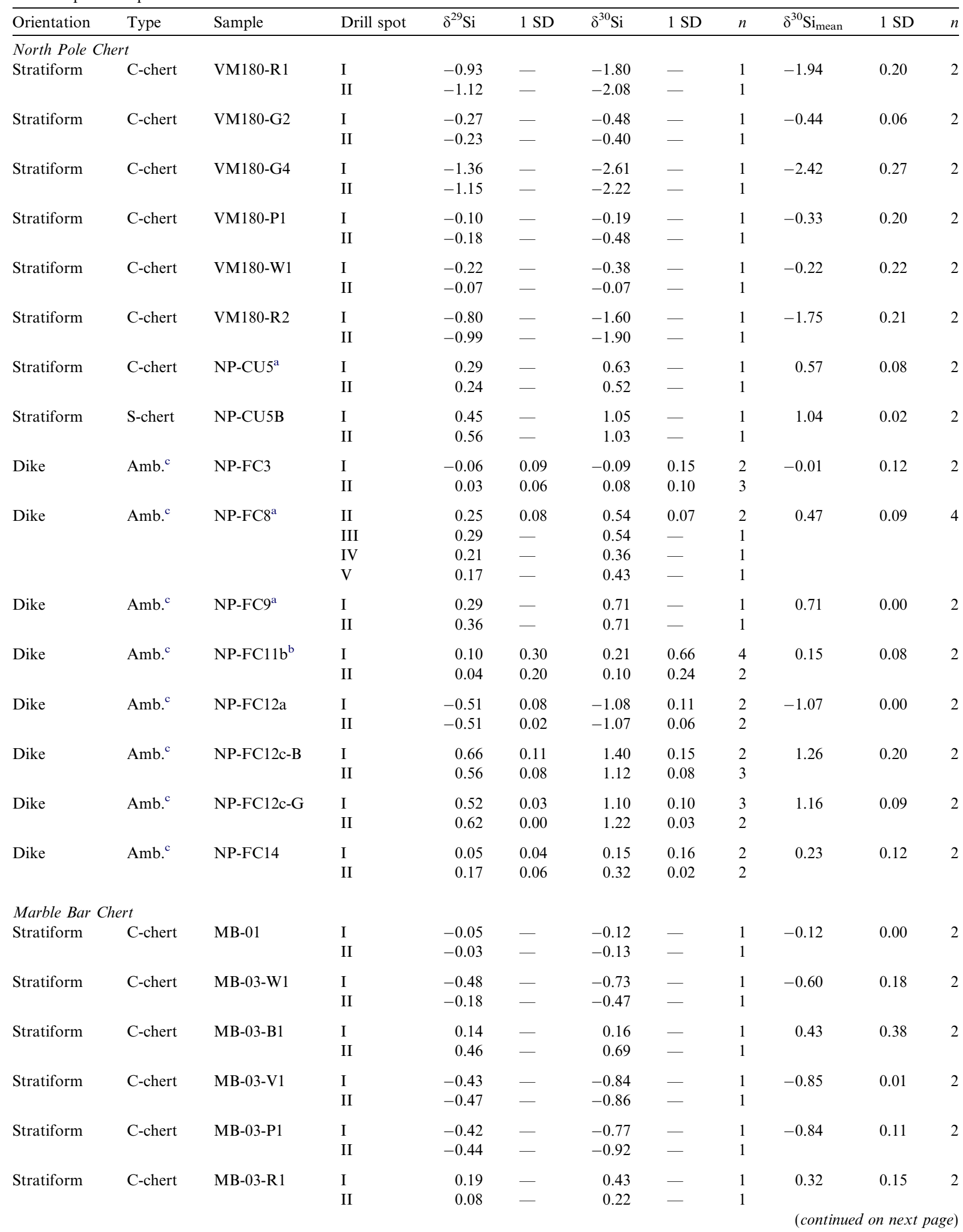


Table 1 (continued)

\begin{tabular}{|c|c|c|c|c|c|c|c|c|c|c|c|}
\hline Orientation & Type & Sample & Drill spot & $\delta^{29} \mathrm{Si}$ & $1 \mathrm{SD}$ & $\delta^{30} \mathrm{Si}$ & $1 \mathrm{SD}$ & $n$ & $\delta^{30} \mathrm{Si}_{\text {mean }}$ & $1 \mathrm{SD}$ & $n$ \\
\hline Stratiform & C-chert & MB-04-W ${ }^{\mathrm{a}}$ & $\begin{array}{l}\text { I } \\
\text { II }\end{array}$ & $\begin{array}{l}-0.27 \\
-0.25\end{array}$ & - & $\begin{array}{l}-0.42 \\
-0.44\end{array}$ & - & $\begin{array}{l}1 \\
1\end{array}$ & -0.43 & 0.02 & 2 \\
\hline Stratiform & C-chert & MB-04-B ${ }^{\mathrm{a}}$ & $\begin{array}{l}\text { I } \\
\text { II }\end{array}$ & $\begin{array}{l}0.22 \\
0.06\end{array}$ & $\begin{array}{l}0.03 \\
0.00\end{array}$ & $\begin{array}{l}0.41 \\
0.16\end{array}$ & $\begin{array}{l}0.02 \\
0.04\end{array}$ & $\begin{array}{l}2 \\
2\end{array}$ & 0.29 & 0.17 & 2 \\
\hline Stratiform & C-chert & MB-06 & $\begin{array}{l}\text { I } \\
\text { II }\end{array}$ & $\begin{array}{l}-0.68 \\
-0.63\end{array}$ & $\begin{array}{l}0.15 \\
0.01\end{array}$ & $\begin{array}{l}-1.34 \\
-1.40\end{array}$ & $\begin{array}{l}0.24 \\
0.04\end{array}$ & $\begin{array}{l}2 \\
2\end{array}$ & -1.37 & 0.04 & 2 \\
\hline Stratiform & C-chert & MB- $09^{\mathrm{a}}$ & $\begin{array}{l}\text { I } \\
\text { II }\end{array}$ & $\begin{array}{l}-0.13 \\
-0.33\end{array}$ & - & $\begin{array}{l}-0.29 \\
-0.60\end{array}$ & - & $\begin{array}{l}1 \\
1\end{array}$ & -0.44 & 0.22 & 2 \\
\hline Stratiform & C-chert & MB-10-R1 & $\begin{array}{l}\text { I } \\
\text { II }\end{array}$ & $\begin{array}{l}-0.22 \\
-0.35\end{array}$ & - & $\begin{array}{l}-0.51 \\
-0.81\end{array}$ & - & $\begin{array}{l}1 \\
1\end{array}$ & -0.66 & 0.21 & 2 \\
\hline Stratiform & C-chert & MB-10-R2 & $\begin{array}{l}\text { I } \\
\text { II }\end{array}$ & $\begin{array}{l}0.26 \\
0.29\end{array}$ & - & $\begin{array}{l}0.59 \\
0.55\end{array}$ & - & $\begin{array}{l}1 \\
1\end{array}$ & 0.57 & 0.03 & 2 \\
\hline Stratiform & C-chert & MB-10-W1 & $\begin{array}{l}\text { I } \\
\text { II }\end{array}$ & $\begin{array}{r}0.03 \\
-0.07\end{array}$ & - & $\begin{array}{r}0.09 \\
-0.12\end{array}$ & - & $\begin{array}{l}1 \\
1\end{array}$ & -0.02 & 0.15 & 2 \\
\hline Stratiform & C-chert & MB-10-Br3 & $\begin{array}{l}\text { I } \\
\text { II }\end{array}$ & $\begin{array}{l}0.13 \\
0.05\end{array}$ & - & $\begin{array}{l}0.31 \\
0.13\end{array}$ & - & $\begin{array}{l}1 \\
1\end{array}$ & 0.22 & 0.12 & 2 \\
\hline Stratiform & C-chert & MB-10-B1 & $\begin{array}{l}\text { I } \\
\text { II }\end{array}$ & $\begin{array}{l}0.32 \\
0.36\end{array}$ & - & $\begin{array}{l}0.52 \\
0.58\end{array}$ & - & $\begin{array}{l}1 \\
1\end{array}$ & 0.55 & 0.04 & 2 \\
\hline Stratiform & Volcanic & APB2 & $\begin{array}{l}\text { I } \\
\text { II }\end{array}$ & $\begin{array}{l}-0.21 \\
-0.08\end{array}$ & - & $\begin{array}{l}-0.28 \\
-0.17\end{array}$ & - & $\begin{array}{l}1 \\
1\end{array}$ & -0.23 & 0.08 & 2 \\
\hline \multicolumn{12}{|c|}{ Kitty's Gap Chert } \\
\hline Stratiform & Volcanic & KG-P1 & $\begin{array}{l}\text { I } \\
\text { II }\end{array}$ & $\begin{array}{l}0.09 \\
0.03\end{array}$ & - & $\begin{array}{l}0.18 \\
0.15\end{array}$ & - & $\begin{array}{l}1 \\
1\end{array}$ & 0.16 & 0.03 & 2 \\
\hline Stratiform & Volcanic & L1-V1 & $\begin{array}{l}\text { I } \\
\text { III }\end{array}$ & $\begin{array}{l}0.15 \\
0.07\end{array}$ & - & $\begin{array}{l}0.36 \\
0.19\end{array}$ & - & $\begin{array}{l}1 \\
1\end{array}$ & 0.28 & 0.12 & 2 \\
\hline Stratiform & C-chert & $\mathrm{L} 1-4.0-\mathrm{G}$ & $\begin{array}{l}\text { I } \\
\text { II }\end{array}$ & $\begin{array}{r}0.00 \\
-0.18\end{array}$ & - & $\begin{array}{r}0.07 \\
-0.26\end{array}$ & - & $\begin{array}{l}1 \\
1\end{array}$ & -0.10 & 0.23 & 2 \\
\hline Stratiform & S-chert & L1-4.0-W & $\begin{array}{l}\text { I } \\
\text { II }\end{array}$ & $\begin{array}{l}0.44 \\
0.44\end{array}$ & - & $\begin{array}{l}0.88 \\
0.87\end{array}$ & - & $\begin{array}{l}1 \\
1\end{array}$ & 0.87 & 0.01 & 2 \\
\hline Stratiform & S-chert & L1-6A-5-B & $\begin{array}{l}\text { I } \\
\text { II }\end{array}$ & $\begin{array}{l}0.42 \\
0.36\end{array}$ & $\begin{array}{l}0.05 \\
-\end{array}$ & $\begin{array}{l}0.86 \\
0.78\end{array}$ & $\begin{array}{l}0.09 \\
-\end{array}$ & $\begin{array}{l}2 \\
1\end{array}$ & 0.82 & 0.05 & 2 \\
\hline Stratiform & S-chert & L1-6A-5-W & $\begin{array}{l}\text { I } \\
\text { II }\end{array}$ & $\begin{array}{l}0.18 \\
0.42\end{array}$ & - & $\begin{array}{l}0.36 \\
0.79\end{array}$ & - & $\begin{array}{l}1 \\
1\end{array}$ & 0.58 & 0.30 & 2 \\
\hline Stratiform & S-chert & L1-8.3 & $\begin{array}{l}\text { I } \\
\text { II }\end{array}$ & $\begin{array}{l}0.40 \\
0.32\end{array}$ & $\begin{array}{l}0.06 \\
-\end{array}$ & $\begin{array}{l}0.87 \\
0.68\end{array}$ & $\begin{array}{l}0.16 \\
-\end{array}$ & $\begin{array}{l}2 \\
1\end{array}$ & 0.78 & 0.13 & 2 \\
\hline Stratiform & S-chert & $\mathrm{L} 1-13.3 \mathrm{~b}-\mathrm{B} 1^{\mathrm{a}}$ & $\begin{array}{l}\text { I } \\
\text { II }\end{array}$ & $\begin{array}{l}0.19 \\
0.11\end{array}$ & - & $\begin{array}{l}0.49 \\
0.30\end{array}$ & - & $\begin{array}{l}1 \\
1\end{array}$ & 0.39 & 0.13 & 2 \\
\hline Stratiform & S-chert & L1-13.3b- $\mathrm{G}^{\mathrm{a}}$ & $\begin{array}{l}\text { I } \\
\text { II }\end{array}$ & $\begin{array}{l}0.10 \\
0.03\end{array}$ & - & $\begin{array}{l}0.30 \\
0.08\end{array}$ & - & $\begin{array}{l}1 \\
1\end{array}$ & 0.19 & 0.16 & 2 \\
\hline Stratiform & S-chert & $\mathrm{L} 1-13.3 \mathrm{~b}-\mathrm{W}^{\mathrm{a}}$ & $\begin{array}{l}\text { I } \\
\text { II }\end{array}$ & $\begin{array}{l}0.15 \\
0.08\end{array}$ & - & $\begin{array}{l}0.27 \\
0.15\end{array}$ & - & $\begin{array}{l}1 \\
1\end{array}$ & 0.21 & 0.08 & 2 \\
\hline Stratiform & S-chert & L1-13.3b-B2 ${ }^{\mathrm{a}}$ & $\begin{array}{l}\text { I } \\
\text { II }\end{array}$ & $\begin{array}{l}0.19 \\
0.20\end{array}$ & - & $\begin{array}{l}0.42 \\
0.39\end{array}$ & - & $\begin{array}{l}1 \\
1\end{array}$ & 0.41 & 0.02 & 2 \\
\hline Stratiform & S-chert & L1-18.7 & $\begin{array}{l}\text { I } \\
\text { II }\end{array}$ & $\begin{array}{l}0.28 \\
0.24\end{array}$ & - & $\begin{array}{l}0.63 \\
0.42\end{array}$ & - & $\begin{array}{l}1 \\
1\end{array}$ & 0.53 & 0.15 & 2 \\
\hline Stratiform & S-chert & L1-52 & $\begin{array}{l}\text { I } \\
\text { II }\end{array}$ & $\begin{array}{l}0.04 \\
0.12\end{array}$ & - & $\begin{array}{l}0.10 \\
0.25\end{array}$ & - & $\begin{array}{l}1 \\
1\end{array}$ & 0.17 & 0.10 & 2 \\
\hline Stratiform & S-chert & L1-73b-G ${ }^{\mathrm{a}}$ & $\begin{array}{l}\text { I } \\
\text { II }\end{array}$ & $\begin{array}{l}0.59 \\
0.43\end{array}$ & $\begin{array}{l}0.07 \\
0.02\end{array}$ & $\begin{array}{l}1.14 \\
0.89\end{array}$ & $\begin{array}{l}0.16 \\
0.03\end{array}$ & $\begin{array}{l}2 \\
2\end{array}$ & 1.01 & 0.18 & 2 \\
\hline
\end{tabular}


Table 1 (continued)

\begin{tabular}{|c|c|c|c|c|c|c|c|c|c|c|c|}
\hline Orientation & Type & Sample & Drill spot & $\delta^{29} \mathrm{Si}$ & $1 \mathrm{SD}$ & $\delta^{30} \mathrm{Si}$ & $1 \mathrm{SD}$ & $n$ & $\delta^{30} \mathrm{Si}_{\text {mean }}$ & $1 \mathrm{SD}$ & $n$ \\
\hline \multirow[t]{2}{*}{ Stratiform } & S-chert & L1-73b-D ${ }^{a}$ & I & 0.56 & - & 1.06 & - & 1 & 1.08 & 0.03 & 2 \\
\hline & & & II & 0.55 & - & 1.10 & - & 1 & & & \\
\hline \multirow[t]{2}{*}{ Stratiform } & S-chert & L1-78b & I & 0.35 & - & 0.87 & - & 1 & 0.87 & 0.01 & 2 \\
\hline & & & II & 0.42 & - & 0.87 & - & 1 & & & \\
\hline \multirow[t]{2}{*}{ Stratiform } & S-chert & $\mathrm{KG}-\mathrm{C} 3^{\mathrm{a}}$ & I & 0.08 & 0.02 & 0.23 & 0.05 & 2 & 0.14 & 0.13 & 2 \\
\hline & & & II & 0.04 & 0.12 & 0.05 & 0.15 & 2 & & & \\
\hline \multirow[t]{2}{*}{ Dike } & S-chert & $\mathrm{KG}-\mathrm{FCl}^{\mathrm{a}}$ & I & 0.45 & - & 0.79 & - & 1 & 0.77 & 0.03 & 2 \\
\hline & & & II & 0.37 & - & 0.76 & - & 1 & & & \\
\hline \multirow[t]{2}{*}{ Dike } & S-chert & KG-FC2a & I & 0.36 & - & 0.69 & - & 1 & 0.53 & 0.22 & 2 \\
\hline & & & II & 0.09 & - & 0.37 & - & 1 & & & \\
\hline \multirow[t]{2}{*}{ Dike } & S-chert. & KG-FC3c-G & I & 0.30 & - & 0.62 & - & 1 & 0.52 & 0.14 & 2 \\
\hline & & & II & 0.13 & - & 0.42 & - & 1 & & & \\
\hline \multirow[t]{2}{*}{ Dike } & S-chert & KG-FC3c-B & I & 0.21 & - & 0.40 & - & 1 & 0.51 & 0.15 & 2 \\
\hline & & & II & 0.29 & - & 0.62 & - & 1 & & & \\
\hline \multirow[t]{2}{*}{ Dike } & S-chert & KG-FC4a & I & 0.43 & - & 0.72 & - & 1 & 0.94 & 0.30 & 2 \\
\hline & & & II & 0.64 & - & 1.15 & - & 1 & & & \\
\hline \multirow[t]{2}{*}{ Dike } & S-chert & KG-FC4b & I & 0.18 & 0.07 & 0.33 & 0.05 & 2 & 0.47 & 0.21 & 2 \\
\hline & & & II & 0.27 & 0.01 & 0.62 & 0.01 & 2 & & & \\
\hline \multirow[t]{2}{*}{ Dike } & S-chert & KG-FC4c-B & I & 0.29 & - & 0.65 & - & 1 & 0.66 & 0.01 & 2 \\
\hline & & & II & 0.33 & - & 0.67 & - & 1 & & & \\
\hline \multirow[t]{2}{*}{ Dike } & C-chert & KG-FC4c-W & I & -0.12 & - & -0.28 & - & 1 & -0.16 & 0.16 & 2 \\
\hline & & & II & -0.02 & - & -0.05 & - & 1 & & & \\
\hline
\end{tabular}

${ }^{a}$ Data from Van den Boorn et al. (2007).

${ }^{\mathrm{b}}$ Multiple analyses of individual solutions from both drill sites result in poor reproducibility $(>0.2 \%, 1 \mathrm{SD})$. The cause is unclear but perhaps not all impurities were separated in this sample; not shown in Fig. 3.

c Type is ambiguous (see text).

convex LREE depletions $\left(0.1<\mathrm{Pr}_{\mathrm{SN}} / \mathrm{Yb}_{\mathrm{SN}}<0.3\right.$, mean $=$ 0.2 ), except for grey lamina VM180-G2. However, REE were close to or below (i.e., Ho and $\mathrm{Tb}$ ) detection limit in this sample. The $\delta^{30} \mathrm{Si}$ of different laminae in sample VM180T is extremely variable with variations of up to $2.2 \%$ (Fig. 3), and $\sim 0.5 \%$ within individual laminae (Table 1; cf., Vroon et al., 2007).

Dike cherts are grey to black and have a microcrystalline quartz groundmass with distinctive accessory mineral assemblages. The black varieties are characterised by a sulphidic assemblage including minor $(\mathrm{Cu}, \mathrm{Zn})$-sulphide, pyrite, galena and Ni-sulphide, and contain clots of carbonaceous material. Occasionally $(\mathrm{Ca}, \mathrm{Mg}, \mathrm{Fe})$ carbonate is observed. Grey cherts generally lack sulphide and accessories are dominated by $\mathrm{Fe}$-(hydr)oxide, barite and Al-sulphate (cf., Ueno et al., 2004). In some of the grey chert samples minor amounts of altered (porous) sericite were observed. Geochemically the black and grey dike cherts are similar to Kitty's Gap dike samples, including variable $\mathrm{Al}_{2} \mathrm{O}_{3}(0.08-1.2 \%)$ and $\mathrm{K}_{2} \mathrm{O}(0-0.2 \%)$ contents. Concentrations of lithophile trace elements fall in the lower range of those at Kitty's Gap: Th (0.03-0.7 ppm), Rb (0.08$6 \mathrm{ppm})$, Hf $(0.02-0.3 \mathrm{ppm}), \mathrm{Ga}(0.2-2 \mathrm{ppm}), \mathrm{Nb}(0.04$ $0.8 \mathrm{ppm})$ and $\mathrm{Zr}(0.8-13 \mathrm{ppm})$. The REE $+\mathrm{Y}$ patterns of different chert samples from a single dike outcrop are remarkably similar although absolute concentrations may vary by an order of magnitude (Fig. 2c and d). Three sam- ples from dike outcrop NP-FC12 are characterised by MREE depletions with relatively flat LREE (from La to $\mathrm{Sm}$ ) and slightly increasing HREE (from Dy to $\mathrm{Lu}$ ). They exhibit subtly positive $\mathrm{Eu}$ anomalies $\left(1.3<\mathrm{Eu} / \mathrm{Eu}_{\mathrm{SN}}^{*}<1.4\right.$, mean $=1.3)$ but lack diagnostic $\mathrm{La}\left(0.9<\mathrm{La} / \mathrm{La}_{\mathrm{SN}}^{*}<1.1\right.$, mean $=1.0)$ and $\mathrm{Y} / \mathrm{Ho}$ anomalies $(25<\mathrm{Y} / \mathrm{Ho}<27$, mean $=26$ ). Three other samples (NP-FC3, NP-FC8 and NP-FC9) taken from a single dike exposure show roughly similar slightly HREE-enriched patterns $\left(0.6<\mathrm{Pr}_{\mathrm{SN}} /\right.$ $\mathrm{Yb}_{\mathrm{SN}}<0.7$, mean $=0.7$ ) with distinct positive Eu anomalies $\left(2.9<\mathrm{Eu} / \mathrm{Eu}_{\mathrm{SN}}^{*}<5.6\right.$, mean $\left.=3.9\right)$ but no $\mathrm{La}(1.1<\mathrm{La} /$ $\mathrm{La}_{\mathrm{SN}}^{*}<1.2$, mean $\left.=1.2\right)$ and $\mathrm{Y} / \mathrm{Ho}$ anomalies $(27<\mathrm{Y} /$ Ho $<29$, mean $=28$ ). Dike samples NP-FC11b and NPFC14 share some characteristics with the Marble Bar Chert samples and sample VM180T. Sample NP-FC11b displays the characteristic convex LREE depletions $\left(\mathrm{Pr}_{\mathrm{SN}} /\right.$ $\left.\mathrm{Yb}_{\mathrm{SN}}=0.2\right)$ and $\mathrm{Eu}$ anomaly $\left(\mathrm{Eu} / \mathrm{Eu}_{\mathrm{SN}}^{*}=1.8\right)$, but lacks the $\mathrm{Y} / \mathrm{Ho}$ anomaly $(\mathrm{Y} / \mathrm{Ho}=25)$. NP-FC14, on the other hand, shows $\mathrm{Y} / \mathrm{Ho}(\mathrm{Y} / \mathrm{Ho}=39)$ and weak $\mathrm{Eu}(\mathrm{Eu} /$ $\left.\mathrm{Eu}_{\mathrm{SN}}^{*}=1.5\right)$ anomalies but lacks the distinct convex LREE depletion $\left(\operatorname{Pr}_{\mathrm{SN}} / \mathrm{Yb}_{\mathrm{SN}}=0.8\right)$. Both samples fail to show a significant La anomaly $\left(\mathrm{La} / \mathrm{La}_{\mathrm{SN}}^{*}=1.1\right)$.

Overall, the North Pole chert-barite unit shows the most extreme variation in $\delta^{30} \mathrm{Si}$ of the three localities, with a total range between $-2.4 \%$ and $+1.3 \%$. Both negative and positive $\delta^{30} \mathrm{Si}$ values are observed with a range of $-2.4 \%$ to $+1.0 \%$ in stratiform chert samples and $-1.1 \%$ o 


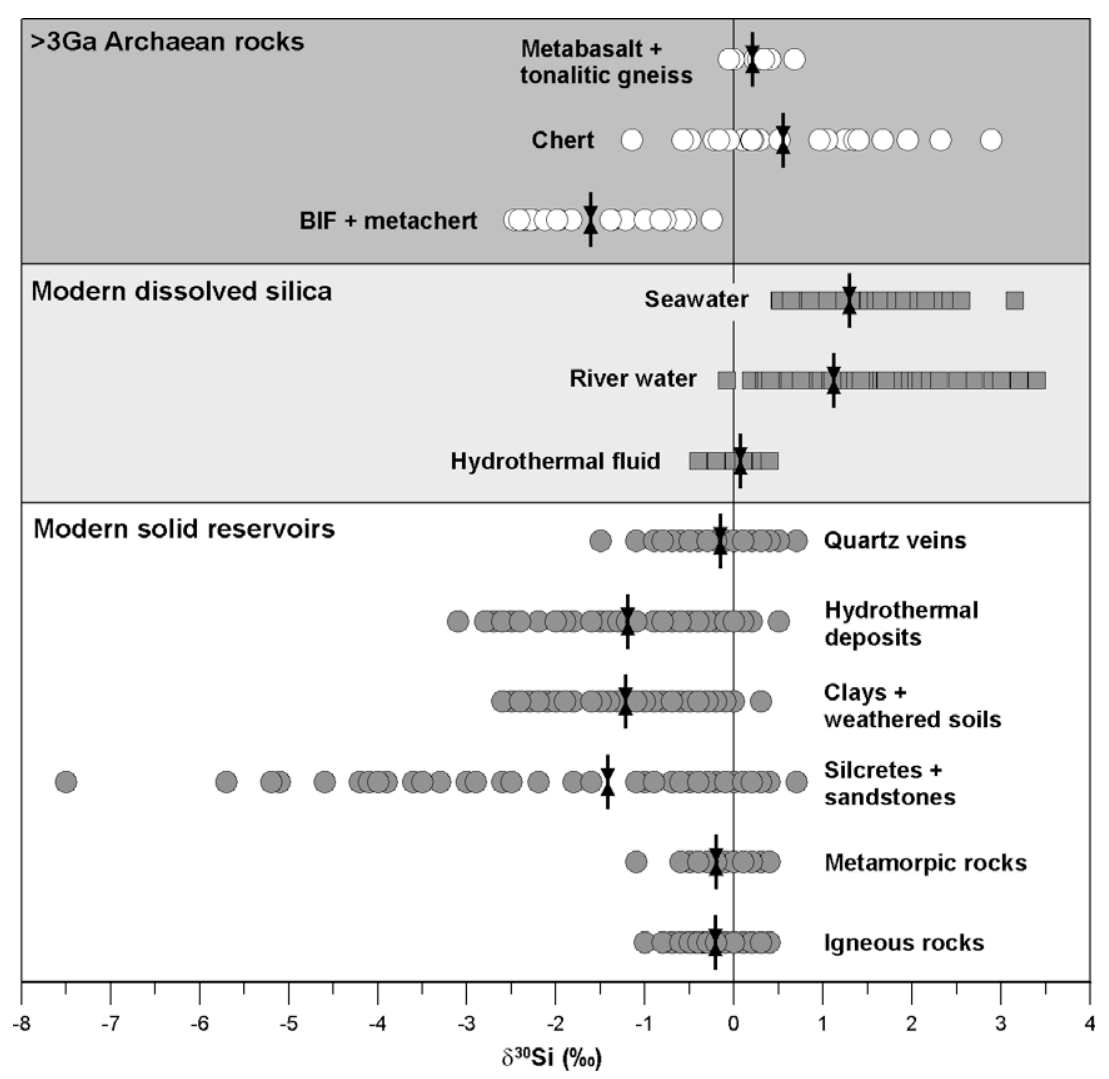

Fig. 4. Silicon isotope compositions of the Earth's main reservoirs (solid or dissolved silica) and $>3.0$ Ga rocks. Arrows indicate the mean values. Data from Douthitt (1982), Ding et al. (1996, 2004), De La Rocha et al. (2000), Basile-Doelsch et al. (2005), Cardinal et al. (2005), Ziegler et al. (2005a,b), André et al. (2006), Robert and Chaussidon (2006), Georg et al. (2006, 2007a,b), Reynolds et al. (2006).

to $+1.3 \%$ in dike cherts. No systematic isotopic offsets were observed between grey and black dike cherts.

\subsection{Volcanic rocks}

The samples from the Apex basalt (APB2) and Panorama felsic volcanic rocks (L1-V1 and KG-P1) are all silicified to a certain extent, resulting in $\mathrm{SiO}_{2}$ contents of $72 \%$, $78 \%$ and $82 \%$, respectively. The felsic nature of the Panorama rocks is evident from the relatively high concentrations of $\mathrm{Zr}(130-140 \mathrm{ppm}), \mathrm{Nb}(6-7 \mathrm{ppm})$ and $\mathrm{Rb}$ (79$99 \mathrm{ppm})$ compared to the Apex Basalt $(\mathrm{Zr}=26 \mathrm{ppm}$, $\mathrm{Nb}=1.3 \mathrm{ppm}$ and $\mathrm{Rb}=0.2 \mathrm{ppm})$. The $\mathrm{REE}+\mathrm{Y}$ pattern of APB2 (not shown) is similar to that of tholeiitic basalt (Kato and Nakamura, 2003), showing strongly depleted LREE (Fig. 2h). The patterns of L1-V1 and KG-P1 (bold lines in Fig. 2e and f, respectively) largely follow the trends found in the Kitty's Gap Chert. Both have minor positive $\mathrm{Eu}\left(1.2<\mathrm{Eu} / \mathrm{Eu}_{\mathrm{SN}}^{*}<1.3\right)$ and $\mathrm{Y} / \mathrm{Ho} \quad(34<\mathrm{Y} / \mathrm{Ho}<36)$ anomalies, and L1-V1 a small negative $\mathrm{Ce}$ anomaly $(\mathrm{Ce} /$ $\mathrm{Ce}_{\mathrm{SN}}^{*}=0.8$ ).

The $\delta^{30} \mathrm{Si}$ value of the silicified Apex Basalt sample $(-0.2 \%)$ is close to the present-day value of basaltic rocks (ca. $-0.3 \%$, e.g., Georg et al., 2007a). The two silicified Panorama volcanic extrusive rocks have slightly higher $\delta^{30} \mathrm{Si}(+0.2 \%$ and $+0.3 \%$ o compared to modern felsic rocks (ca. $-0.1 \%$ ) (Ding et al., 1996).

\section{DISCUSSION}

\subsection{Mineralogical and geochemical distinction between 'silicified precursor chert' and 'chemical chert'}

Two fundamental mechanisms can be invoked to explain chert formation in the Archaean: transformation of a precursor lithology (S-chert) or orthochemical precipitation (C-chert) from a silica-rich fluid (Van den Boorn et al., 2007). Although diagnostic features are not always conclusive, macroscopic features and/or mineralogical and geochemical data generally provide a solid basis to distinguish between these processes. The hypothesis that secondary alteration processes play a role was discounted by Van den Boorn et al. (2007) and will be discussed in more detail in Section 5.5.

\subsubsection{Silicified precursor chert ( $S$-chert)}

Precursor lithologies of S-cherts are diverse. The extent of silicification may pose limits to their identification and distinction from C-cherts. In general, two main types of Archaean replacement cherts have been documented. Evaporatic precursors (Buick and Dunlop, 1990; Sugitani et al., 1998; Lowe and Fisher Worrell, 1999) are commonly recognised by preserved silicified pseudomorphs of carbonate, gypsum and/or nahcolite and/or by remains of these precursor minerals. Replacement of volcanogenic sediments 
(DiMarco and Lowe, 1989a; Sugitani et al., 1996, 1998; Lowe, 1999; De Vries, 2004; Orberger et al., 2006) is generally characterised by impurities of sericite/chlorite, Ti-oxides and zircons. Obvious sedimentary structures are occasionally preserved and provide evidence for settling of volcanic detritus directly from the water column or reworking of sediments in a shallow marine environment (DiMarco and Lowe, 1989b; Nijman et al., 1999b; De Vries, 2004). Silicification of solid extrusive rocks is an equivalent process but is often less intense $\left(<90 \% \mathrm{SiO}_{2}\right)$, presumably due to lower permeability.

Stratiform chert samples from the Kitty's Gap Chert bear all the petrographic characteristics of a replaced primary or reworked volcanogenic deposit. Abundant sericites are likely alteration products of feldspars, while zircon, Ti-oxide, detrital quartz, monazite and apatite are residues of the precursor assemblage that survived pervasive silicification. Preserved sedimentary structures point to a shallowmarine origin of the Kitty's Gap Chert (cf., Nijman et al., 1999b; De Vries, 2004).

The mineralogical data point to a largely felsic volcanogenic precursor (cf., DiMarco and Lowe, 1989a; Lowe, 1999; Rouchon and Orberger, 2008), in accordance with locally preserved pumiceous fragments (Westall et al., 2006). The presence of $(\mathrm{Fe}, \mathrm{Cr}$ )-oxides (spinels?) and higher $\mathrm{Cr}$ contents in greenish chert layers towards the top of the sequence hint at an (ultra)mafic component (cf., Lowe, 1999). The precursor was probably not exclusively ultramafic, given the occasional presence of sericite and zircon. Hence, volcanogenic sedimentation was most likely dominated by felsic material in the lower stratigraphic levels, with an (ultra)mafic source becoming more pronounced towards the top.

The REE +Y signatures of the Kitty's Gap Chert samples confirm the large contribution of felsic detritus (cf., Orberger et al., 2006; Rouchon and Orberger, 2008). MUQ-shale-normalised patterns are relatively flat, showing slight LREE or HREE enrichments, and are broadly similar to the patterns of unaltered felsics from the Duffer Formation (Fig. 2h). Minor positive Eu anomalies suggest that precursor volcaniclastics were slightly more enriched in plagioclase than MUQ shale. Taking the large contribution of basaltic material in MUQ shale (Bolhar et al., 2005) into account, it is conceivable that the Kitty's Gap volcaniclastic rocks were mainly derived from erosion of the underlying felsic Duffer (or perhaps Panorama) Formation, which also shows small positive $\mathrm{Eu}$ anomalies when normalised to MUQ (Fig. 2e, f and h). The variable slopes of REE+Y patterns and relative LREE depletions in some of the Kitty's Gap Cherts might be due to admixture of mafic components or to other variations in the origin of precursor materials.

Partial overprinting of original geochemical signatures by seawater must be considered as an alternative or superimposed process responsible for variations in the REE $+Y$ patterns. It might explain the LREE depletions, the negative $\mathrm{Ce}$ anomalies (if seawater was locally oxygenated; cf., Orberger et al., 2006), as well as the slightly higher Y/Ho ratios relative to the chondritic value of $\sim 26$ in some of the Kitty's Gap samples (Fig. 2e and f). However, Ce anomalies have also been attributed to post-depositional alteration and might therefore not be diagnostic of seawater (Hayashi et al., 2004). Positive La anomalies, another seawater signal, are absent but could have been suppressed by the high LREE concentrations of crustal precursor components. We thus conclude that REE+Y patterns of Kitty's Gap Chert samples are largely determined by the felsic precursor. Its overall high REE $+\mathrm{Y}$ content masks unequivocal seawater signatures which are weak or absent.

Many dike cherts from the Kitty's Gap Chert are also characterised by elevated $\mathrm{Al}_{2} \mathrm{O}_{3}$ and $\mathrm{K}_{2} \mathrm{O}$ contents and the presence of sericite, zircon and Ti-oxide. The broad similarity in REE $+Y$ patterns with stratiform cherts suggests that the dikes contain silicified sediments that slumped down from the surface. This is supported by the presence of sedimentary chert clasts below the palaeosurface (i.e., the stratiform chert). However, it seems unlikely that infilling by sediments occurred all the way downwards into the finely ramified basal parts of dikes or veins $>100 \mathrm{~m}$ below the palaeosurface. We therefore propose that at least part of the dike cherts represents chert-cemented silicified wall rock of the felsic Panorama Formation (cf., Nijman et al., 1999b, their Fig. 11a).

The silicified-sandstone sample (NP-CU5) from the stratiform North Pole deposit probably had an (ultra)mafic precursor, which is consistent with the absence of felsic volcanic rocks in the underlying successions. The sample lacks sericites and zircons but contains Fe-Mg silicates. The LREE-depleted pattern with relatively flat HREE (Fig. 2d) is similar to unsilicified ultramafic rocks from the Coonterunah and Apex Formation (Fig. 2h). The modest $\mathrm{Eu}$ anomaly seems incompatible with a felsic component and may reflect partial overprinting by a hydrothermal fluid (see below).

The origin of the North Pole dike cherts is more ambiguous and will be discussed separately in Section 5.1.3.

\subsubsection{Chemical chert (C-chert)}

We use the term C-chert for deposits formed by direct orthochemical precipitation of silica from Si-rich fluids. Almost pure white silica bands and ferruginous cherts are generally believed to represent orthochemical deposits (cf., Knauth and Lowe, 2003; Van Kranendonk, 2006). Their accessory mineral assemblage is dominated by Fe-(hydr)oxides and minor sulphides and lacks diagnostic precursor minerals. Geochemical signatures are sometimes more diagnostic, because visual inspection may not be sufficiently adequate to rule out a replacement origin in cases of pervasive silicification. Pure chemical precipitates have been distinguished from silicified volcanogenic deposits by strong depletions in lithophile elements and by distinct REE $+\mathrm{Y}$ patterns (Bau and Dulski, 1996; Van Kranendonk et al., 2003; Bolhar et al., 2004, 2005; Friend et al., 2008; Alexander et al., 2009). Some of these features, however, may also apply to cherts that replace carbonates (or gypsum). This issue is discussed in more detail in Section 5.5.

All Marble Bar Chert samples and sample VM180T from the North Pole Chert have low concentrations of lithophile elements and show variably positive $\mathrm{Eu}, \mathrm{Y}$ and La anomalies as well as $\operatorname{Pr}_{\mathrm{SN}} / \mathrm{Yb}_{\mathrm{SN}}<1$. Positive $\mathrm{Y}$ and 
La anomalies and convex LREE depletions are generally attributed to a seawater source, whereas Eu anomalies reflect the contribution of high-temperature $\left(>250{ }^{\circ} \mathrm{C}\right)$ hydrothermal fluids (Bau and Dulski, 1996; Van Kranendonk et al., 2003; Bolhar et al., 2004, 2005; Friend et al., 2008). Consequently, the samples with positive Y, La and/or Eu anomalies can be interpreted as precipitates from seawater, hydrothermal fluids or mixtures thereof. As illustrated in Fig. 2a and b, the Marble Bar Cherts and the distinct layers in sample VM180T bear a close resemblance to the REE+Y patterns previously reported for cherts, BIF's and carbonates from the Pilbara and Isua greenstone belts that have been attributed to orthochemical precipitation, mostly from seawater, which is inferred to have a positive Eu anomaly due to enhanced hydrothermal inputs in the Archaean (Fig. 2g). Preservation of the diagnostic anomalies demonstrates that detrital input was minor or absent.

The REE $+Y$ patterns of two patches of virtually pure silica in a stratiform and dike chert from Kitty's Gap show some (though not all) of the aforementioned characteristics, which, together with low concentration of lithophile elements, points to orthochemical precipitation.

\subsubsection{Ambiguous dike chert samples from the North Pole Chert}

It is difficult to unequivocally assign our dike cherts from the North Pole locality to one of the genetic groups. Their mineralogical composition would largely define them as C-cherts but slightly elevated lithophile-element concentrations suggest involvement of crustal components. We note that care should be taken to exclusively use $\mathrm{Al}$ as a proxy for crustal inputs because of the presence of minor amounts of Al-sulphates in some of the dike chert samples.

The REE $+Y$ patterns are variable and do not match typical C- or S-chert signatures, although they are remarkably similar in multiple samples from a given dike. For example, different samples from dike NP-FC12 all show a peculiar MREE depletion (Fig. 2c). Three samples from another dike (NP-FC3, NP-FC8 and NP-FC9) are all characterised by relatively flat $\mathrm{REE}+\mathrm{Y}$ patterns but with pronounced positive Eu anomalies (Fig. 2d). The size of the $\mathrm{Eu}$ anomalies and absence of HREE depletions make it difficult to attribute these signatures to felsic (plagioclase-rich) precursor material (Fig. 2h). Instead, they resemble the REE + Y pattern of a siderite sample (code 177886) from the Panorama Formation described by Van Kranendonk et al. (2003) and interpreted as a hydrothermal deposit. Samples NP-FC11b and NP-FC14 from two other dikes have $\mathrm{REE}+\mathrm{Y}$ characteristics in common with $\mathrm{C}$-cherts but also show certain differences as explained in Section 4.3.

Earlier studies are also inconclusive as to the origin of dike cherts from North Pole. Some suggested that the dikes represent barite and silica infillings related to hydrothermal activity (Nijman et al., 1999a; Ueno et al., 2004), whereas others inferred a precursor clay lithology (Orberger et al., 2006). Since our mineralogical and geochemical data are not sufficiently diagnostic, we label the North Pole group of samples as 'ambiguous'. Nonetheless, the strong variability in REE $+Y$ patterns of these chert dikes points to nonuniformity in genetic processes. Instead, they likely reflect mixtures of heterogeneous sediments, wallrock and chemically precipitated silica, presumably in different proportions.

\subsection{Silicon isotope systematics in Early Archaean cherts}

Silicon isotope compositions of the C- and S-chert samples are clearly distinct (Fig. 3 and Table 1). The C-cherts cover a wide range $\left(\delta^{30} \mathrm{Si}=-2.4 \%\right.$ o to $\left.+0.6 \%\right)$, whereas all S-cherts have positive $\delta^{30} \mathrm{Si}$ values showing a much more restricted range $(+0.1 \%$ to $+1.1 \%)$. Thus, despite some overlap, the C-cherts are more depleted in ${ }^{30} \mathrm{Si}$ (lower in $\delta^{30} \mathrm{Si}$ ) than S-cherts. The two types further differ in the extent of isotopic inhomogeneity on hand-specimen scale. Inter-lamina variability on $\mathrm{cm}-\mathrm{mm}$ scale is much larger in C-chert specimen. Poorer reproducibility of data from separate drill points also reflects isotopic heterogeneity within individual laminae and is not attributable to analytical error. In contrast, isotopic heterogeneity between individual laminae and between clasts and groundmass of S-chert specimens is small and often falls within analytical precision.

Differences between the two chert types are illustrated in Fig. 5 where diagnostic $\mathrm{REE}+\mathrm{Y}$ anomalies are plotted against $\delta^{30} \mathrm{Si}$. Stratiform C-cherts span a range in $\delta^{30} \mathrm{Si}$ and are characterised by positive $\mathrm{Y} / \mathrm{Ho}$ and Eu anomalies (as well as positive La anomalies, not shown), whereas stratiform S-cherts cluster at positive $\delta^{30} \mathrm{Si}$ values and are devoid of anomalous REE $+\mathrm{Y}$ abundances. The Kitty's Gap dike chert samples have positive $\delta^{30} \mathrm{Si}(+0.5 \%$ to $+1.0 \%$ ) and largely plot in the field of the stratiform Scherts. Only two of the Kitty's Gap samples (L1-4.0-G and $\mathrm{KG}-\mathrm{FC} 4 \mathrm{c}-\mathrm{W}$ ) have slightly negative $\delta^{30} \mathrm{Si}$ values $(-0.1 \%$ to $-0.2 \%)$ that plot in the field of C-cherts. On the other hand, the ambiguous North Pole dike samples display considerable scatter and have variable $\delta^{30}$ Si ranging from negative to the most positive values found in this study $(-1.1 \%$ to $+1.3 \%)$.

Two mechanisms can be envisaged to explain the Si isotope variability in Early Archaean chert samples from the Pilbara. First, the inhomogeneity reflects mixing between various sources of silica with distinct isotopic signatures (cf., Van den Boorn et al., 2007). We will refer to this option as the 'end-member mixing model'. Alternatively, fractionation mechanisms during silica deposition from silica-bearing fluids account for the observed variation. This explanation will be referred to as the 'fractionation model'. Below the two models will be explored separately, without implying that they are mutually exclusive.

\subsubsection{Mixing model}

A $\delta^{30} \mathrm{Si}$ vs. $\mathrm{Al}_{2} \mathrm{O}_{3}$ diagram (Fig. 6) illustrates that $\mathrm{Si}$ isotope variability in Early Archaean cherts from the Pilbara can be largely explained by mixing of three main sources of silica: (1) hydrothermal fluids; (2) silica-saturated seawater; and (3) volcanic material (Van den Boorn et al., 2007). Stratiform C-cherts fall on the $\mathrm{Al}_{2} \mathrm{O}_{3}$-poor side of the graph $(<0.2$ wt. $\%)$, whereas stratiform S-cherts are enriched in $\mathrm{Al}_{2} \mathrm{O}_{3}(>0.7$ wt. $\%)$.

The $\mathrm{Al}_{2} \mathrm{O}_{3}$-rich end-member approximates primary igneous material as it has a $\delta^{30} \mathrm{Si}$ value close to or slightly 
(a)

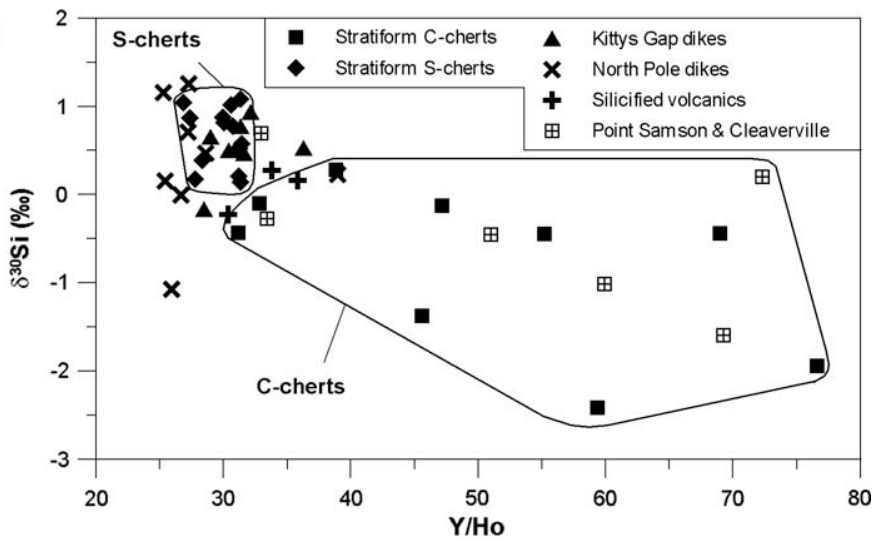

(b)

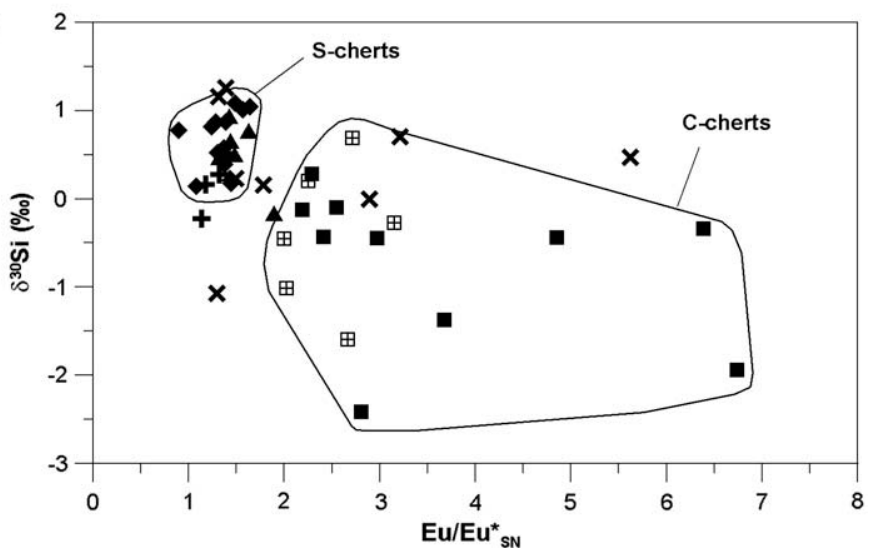

Fig. 5. $\delta^{30} \mathrm{Si}$ vs. (a) $\mathrm{Y} / \mathrm{Ho}$ and (b) $\mathrm{Eu} / \mathrm{Eu}_{\mathrm{SN}}^{*}$. Stratiform C-cherts (filled squares) are characterised by strong Si isotope variation and anomalous $\mathrm{Y} / \mathrm{Ho}$ and $\mathrm{Eu} / \mathrm{Eu}_{\mathrm{SN}}^{*}$; stratiform S-cherts (filled diamonds) show a restricted $\delta^{30} \mathrm{Si}$ range and terrestrial/chondritic $\mathrm{Y} / \mathrm{Ho}(\sim 26)$ and $\mathrm{Eu} / \mathrm{Eu}_{\mathrm{SN}}^{*}$. Dikes from Kitty's Gap (filled triangles) largely fall in the range of the stratiform S-cherts pointing to a genetic relationship. North Pole dike cherts (crosses) show more scatter, especially in $\mathrm{Eu} / \mathrm{Eu}_{\mathrm{SN}}^{*}$. Stratiform C-cherts from Point Samson and Cleaverville (squares with cross) are plotted for comparison (data from Van den Boorn et al., 2007).

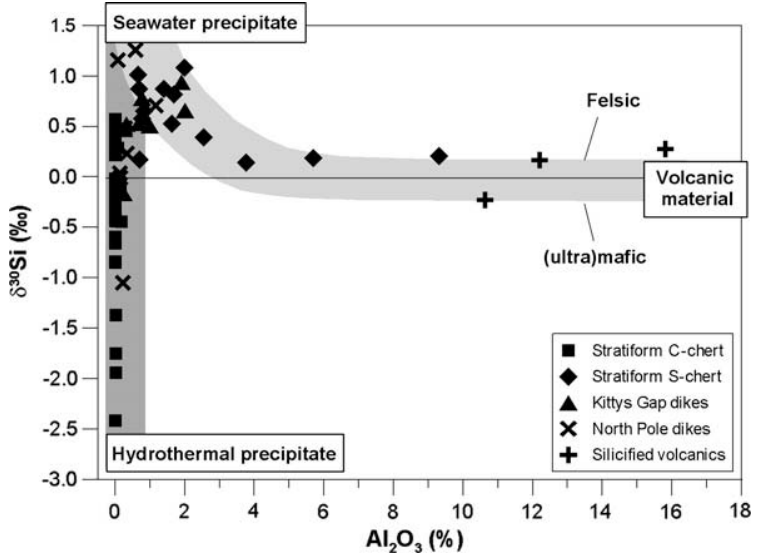

Fig. 6. $\mathrm{Al}_{2} \mathrm{O}_{3}$ content vs. $\delta^{30} \mathrm{Si}$ of chert samples. Subdivision into stratiform C- and S-cherts is given in Tables 1 and EA-2. Dike cherts from Kitty's Gap and North Pole and silicified volcanics are shown separately. S-chert samples fall within the light-grey band that defines mixing between mafic or felsic volcanogenic sediments and seawater. Dark-grey $\delta^{30} \mathrm{Si}$ array represents mixing between hydrothermal fluids and seawater, or evolution of cooling hydrothermal fluids (see text for explanation). below $0 \%$. The low $-\mathrm{Al}_{2} \mathrm{O}_{3}$ cherts plotting between the two other end-members can be interpreted as silica precipitates from mixtures of ${ }^{30} \mathrm{Si}$-enriched and ${ }^{30} \mathrm{Si}$-depleted fluids (Van den Boorn et al., 2007). These fluids were probably isotopically heavier than the cherts, given the general trend that deposits are isotopically lighter than co-existing fluids (e.g., clays and silcretes vs. groundwater, diatoms/sponges vs. seawater; adsorbed silica on Fe-oxides vs. parent water; Ziegler et al., 2005a,b; Basile-Doelsch et al., 2005; De La Rocha et al., 1997; De La Rocha, 2003; Georg et al., 2006, 2007b; Delstanche et al., 2009). In fact, our low $\delta^{30} \mathrm{Si}$ end-member falls within the range of modern hydrothermal silica deposits (Fig. 4).

The ${ }^{30} \mathrm{Si}$-enriched end-member approximates the isotopic composition of modern seawater (Fig. 4; De La Rocha et al., 2000; Cardinal et al., 2005; Reynolds et al., 2006), the positive $\delta^{30} \mathrm{Si}$ of which is mainly the result of the preferential uptake of ${ }^{28} \mathrm{Si}$ by diatoms (De La Rocha et al., 1997) and the continuous supply of isotopically heavy river waters (De La Rocha et al., 2000; Ding et al., 2004; Georg et al., 2006, 2007b). However, silica-secreting organisms had not yet evolved in the Archaean, allowing silica concentration to maintain levels around the saturation of amorphous silica (e.g., Siever, 1992). Continuous supply of silica to these saturated oceans 
by hydrothermal activity and perhaps continental weathering must eventually have led to inorganic removal of silica by precipitation and/or sorption processes. Hence, since silica precipitation will result in relative ${ }^{30} \mathrm{Si}$-enrichment in remaining water, it is reasonable to assume that Archaean oceans had positive $\delta^{30} \mathrm{Si}$ signatures as well (Van den Boorn et al., 2007). The degree of enrichment may have been critically dependent on the temperature difference between seawater and hydrothermal fluids (Robert and Chaussidon, 2006), an issue that will be discussed in Section 5.2.2. Despite the negative sign of precipitation-associated fractionation, cherts with a major seawater component can still be positive. For example, $\delta^{30}$ Si values up to $+1.9 \%$ have been reported in diatom mats from the Central North Pacific (De La Rocha et al., 2000). The positive $\delta^{30} \mathrm{Si}$ values of several of the Ccherts thus imply that $\delta^{30} \mathrm{Si}$ of Archaean seawater should have been even more positive, presumably with a lower limit of at least ca. $+1.3 \%$, the maximum value found in the entire set of all chert types. The actual offset will depend on the applicable average magnitude of fractionation during precipitation.

5.2.1.1. Volcanogenic precursor chert. Our Si isotope results point to seawater as the dominant silicifying medium at Kitty's Gap, in line with the preservation of (weak) seawater-like REE $+\mathrm{Y}$ patterns in some of the samples (Fig. 2e and $\mathrm{f}$ ). Up to $1 \%$ variability in $\delta^{30} \mathrm{Si}$ of the stratiform $\mathrm{S}$ cherts largely reflects differences in the degree of silicification, as is illustrated in Fig. 6. Cherts with the highest $\mathrm{Al}_{2} \mathrm{O}_{3}$ contents plot at low $\delta^{30} \mathrm{Si}$ values, consistent with a large proportion of volcanogenic precursor material with a more ${ }^{30} \mathrm{Si}$-depleted signature relative to seawater. Progressively lower $\mathrm{Al}_{2} \mathrm{O}_{3}$ contents, signalling more pervasive silicification, are accompanied with an increase in $\delta^{30} \mathrm{Si}$ that corresponds to increased additions of silica from ${ }^{30} \mathrm{Si}$-enriched seawater. Non-uniform silicification at Kitty's Gap is consistent with field evidence (De Vries, 2004).

Several factors can be envisaged to explain the heterogeneous nature of silicification processes at Kitty's Gap: (a) fluctuating deposition rates of sediments influenced the duration of their exposure to silica-saturated seawater and thus the water/sediment ratios during interaction, (b) variations in the silica concentration of local (shallow) seawater, possibly induced by evaporation, (c) variations in permeability and porosity, determined by sorting and grain size, which would also influence effective water/sediment ratios. Some support for this last explanation comes from sample L1-13.3b wherein light-coloured laminae are coarser grained and less silicified than black layers. In addition, fine-grained layers (ash or dust) at the top of the unit show the highest $\delta^{30} \mathrm{Si}$ and lowest $\mathrm{Al}_{2} \mathrm{O}_{3}$ contents. Alternatively, more pervasive silicification, resulting in high $\delta^{30} \mathrm{Si}$, reflects better sorting of sediments. Such a presumed relationship between grain size and silicification is less obvious for the single stratiform S-chert sample from the North Pole Chert, considering its rather high $\delta^{30} \mathrm{Si}$ value $(\sim 1.0 \%$ o $)$ and coarse grain size. However, the precursor sediment of this chert was probably (ultra)mafic in composition (Section 5.1.1), making it more susceptible to alteration by seawater (see below).
A final parameter that potentially plays a role in determining the Si isotopic composition of Archaean S-cherts is the precursor composition. The light-grey band in Fig. 6 schematically defines mixing between two hypothetical precursor materials and the ${ }^{30} \mathrm{Si}$-enriched fluid reservoir. The lower limit passes through the silicified Apex Basalt, which has a relatively low $\delta^{30} \mathrm{Si}$ value and low $\mathrm{Al}_{2} \mathrm{O}_{3}$ content, whereas the upper limit reflects the higher $\delta^{30} \mathrm{Si}$ and more aluminous nature of felsic rocks from the Panorama Formation. Although $\delta^{30} \mathrm{Si}$ offset between felsic and mafic rocks (and thus the theoretical width of the band) is ill-constrained (cf., Douthitt, 1982; Ding et al., 1996; Georg et al., 2007a), it is of interest to note that virtually all of our stratiform chert samples plot within the field defined by the two mixing curves.

In summary, the mixing model predicts that isotopic variability in S-cherts is largely determined by the extent of silicification. Minor differences in the isotopic composition of the precursor probably add to the observed heterogeneity.

5.2.1.2. Chemical chert. The $\delta^{30} \mathrm{Si}$ array displayed by the C-cherts (Fig. 6) can be interpreted as the result of mixing between hydrothermally derived (lowest $\delta^{30} \mathrm{Si}$ ) and seawater silica (Van den Boorn et al., 2007). Silica precipitation near modern seafloor hydrothermal venting sites does not occur in volumes comparable to those inferred for the Early Archaean, which points to fundamental differences with the present-day situation (e.g., Siever, 1992). In order to explore apparent discrepancies we first review some of the basic principles of silica behaviour in hydrothermal settings.

5.2.1.3. Silica in hydrothermal fluids and seawater. The silica concentration of hydrothermal fluids is largely governed by equilibration with quartz at high temperature $(\sim 200$ $400{ }^{\circ} \mathrm{C}$ ) and pressure (100-500 bars) in the reaction zone of convection cells (Von Damm et al., 1985, 1991). Hydrothermal fluids become enriched in silica at depth, because its solubility increases with temperature and pressure. Rapid drops in temperature and pressure en route to the surface result in oversaturation with respect to silica, but sluggish precipitation kinetics generally prevent major instant losses of dissolved silica. This conservative behaviour of silica is one of the fundaments of fluid geothermobarometry (Fournier, 1985; Von Damm et al., 1991).

Slow precipitation of quartz under near-surface conditions promotes deposition of amorphous silica instead, which is an ubiquitous phase in sinter deposits around continental hydrothermal springs (e.g., Rimstidt and Cole, 1983; Rodgers et al., 2004). To reach saturation of amorphous silica, temperature and pressure drops must be sufficiently large because it is over an order of magnitude more soluble than quartz. The solubility curve for amorphous silica, shown in Fig. 7 and valid for distilled water at $P_{\text {sat }}$ in the range $0-350{ }^{\circ} \mathrm{C}$ (Gunnarsson and Arnorsson, 2000), is taken to be representative of amorphous silica solubility in seawater at low pressures (cf., Krauskopf, 1956; Robert and Chaussidon, 2006).

Silica concentrations in deep hydrothermal fluids that are controlled by quartz are more variable. Fig. 7 shows 


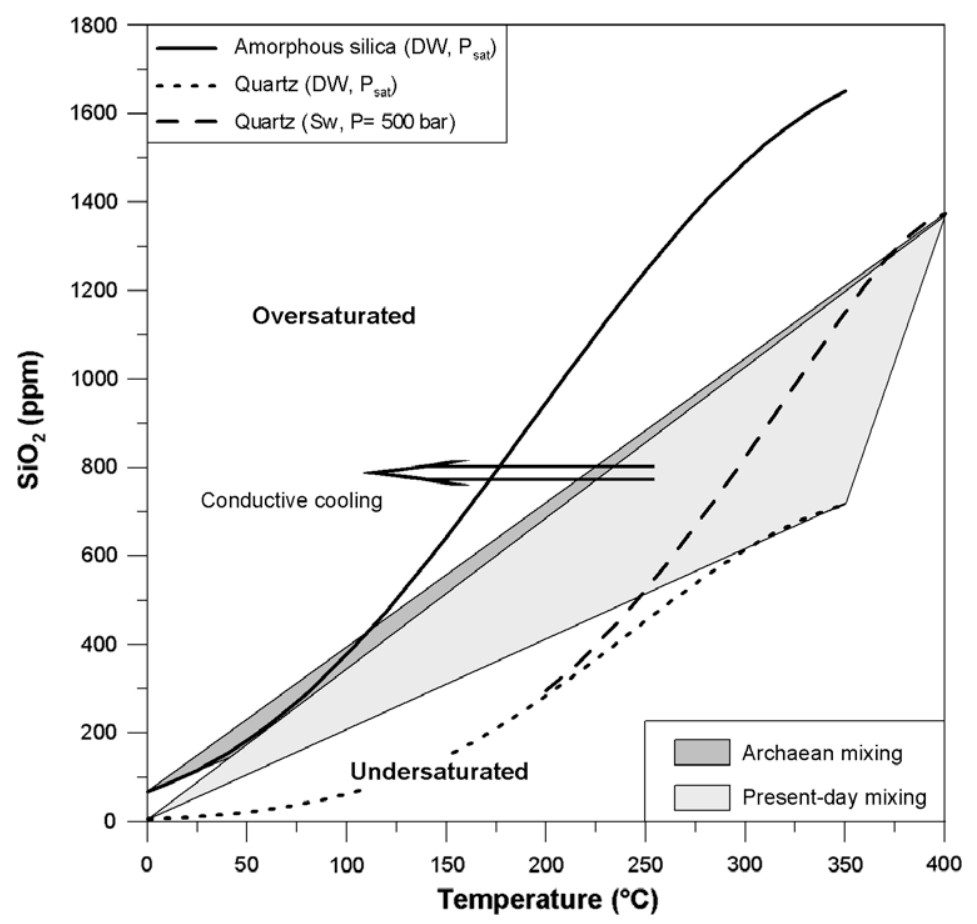

Fig. 7. Temperature-dependent solubility curves for amorphous silica (solid line) and quartz (dotted line) in distilled water (DW) from 0 to $350{ }^{\circ} \mathrm{C}$ at $P_{\text {sat }}$ (Gunnarsson and Arnorsson, 2000) and for quartz in seawater (SW; dashed line) from 0 to $400{ }^{\circ} \mathrm{C}$ at $P=500$ bars (Von Damm et al., 1991; using temperature and pressure-dependent equation for seawater density from Bischoff and Rosenbauer, 1985). Grey fields indicate potential mixing between seawater and hydrothermal fluids for present-day and Archaean conditions (see text for explanation). Large arrow indicates schematic pathway for conductive cooling of hydrothermal fluids.

separate curves for the solubility of quartz in distilled water at $P_{\text {sat }}$ (Gunnarsson and Arnorsson, 2000) and in seawater at $P=500$ bars (Von Damm et al., 1991) to visualise the effect of salt and pressure in the high-temperature domain. At low temperatures the solubility difference is negligible but above $T \sim 200^{\circ} \mathrm{C}$ the curves deviate. A pressure of 500 bars is assumed to be the maximum at which fluids equilibrate with quartz in hydrothermal systems. Realistic silica concentrations in hydrothermal fluids will lie between the two solubility curves (see below).

5.2.1.4. Mixing between hydrothermal fluids and seawater. Fig. 7 compares the effects of mixing between hydrothermal fluid and seawater under modern and Archaean conditions. The principle difference stems from the concentration of silica in seawater. Present-day silica-undersaturated seawater is plotted at $\sim 0{ }^{\circ} \mathrm{C}$ (i.e., close to deep ocean bottom water of $\sim 4{ }^{\circ} \mathrm{C}$ ), whereas Archaean seawater is taken to be saturated with amorphous silica at the same temperature. Because the properties of hydrothermal water are illconstrained, two different end-members are considered. One has a high silica concentration corresponding to the solubility of quartz in seawater at $T \sim 400{ }^{\circ} \mathrm{C}$ and $P$ $\sim 500$ bar, roughly the most extreme temperature and pressure regime in typical hydrothermal convection cells at MOR settings (Seyfried, 1987). The other has a low silica concentration, for which we arbitrarily adopt the minimum concentration in distilled water in equilibrium with quartz at $350{ }^{\circ} \mathrm{C}$ and $P_{\text {sat }}$. As will be discussed in the next section, hydrothermal fluids in the Archaean might have carried even less silica.

Taking this range of hypothetical hydrothermal endmembers into account, and ignoring the temperaturedependent specific heat capacity of water, Fig. 7 shows fields of potential mixtures for modern and Archaean scenarios. Because a mixture of modern seawater and any hydrothermal fluid lies below the solubility curve of amorphous silica, mixing will generally not induce precipitation, which explains the common paucity of amorphous silica deposition in modern seafloor hydrothermal systems (Janecky and Seyfried, 1984). Occasional deposition can be due to strong conductive cooling of a fluid (arrow in Fig. 7) instead (e.g., Stüben et al., 1994; Kristall et al., 2006). In contrast, mixing between hydrothermal fluid and silica-saturated Archaean ocean water could have resulted in precipitation of silica, as the S-shaped solubility curve of amorphous silica is crossed even for a hydrothermal end-member with relatively low silica concentrations.

However, there are a few points to note. Firstly, rather large amounts of seawater would be required to induce amorphous silica precipitation by simple mixing, which seems difficult to reconcile with observed $\delta^{30} \mathrm{Si}$ values as low as $-2.4 \%$, despite the up to ten times higher silica concentrations in hydrothermal fluids. Such values would be more in line with precipitation of silica from rather pristine hydrothermal fluids after a significant drop in temperature by conductive cooling. Secondly, the predicted field of oversaturation strongly depends on the adopted seawater tem- 
perature. If Archaean seawater temperatures were higher than the assumed minimum of $0{ }^{\circ} \mathrm{C}$ (see discussion below), the field of oversaturation - and thus the likelihood of silica precipitation - would reduce considerably. Hence, in order to explain the massive accumulation of silica in the Archaean, it seems reasonable to consider a role for conductive cooling.

\subsubsection{Fractionation model}

As an alternative for mixing with seawater, it is conceivable that hydrothermal fluids produced the entire $\delta^{30} \mathrm{Si}$ range through progressive losses of fractionated silica due to conductive cooling. Here we explore a fractionation model, focusing first on input parameters and then on a possible application to chert formation.

5.2.2.1. Input parameters for fractionation model. Changes in the isotopic composition of an evolving hydrothermal fluid and its co-genetic deposits will depend on: (1) the initial composition of the fluid $\left(\delta^{30} \mathrm{Si}_{\text {initial }}\right)$, and (2) the fractionation factor associated with precipitation (expressed as $\Delta_{\text {solid-fluid }}$; i.e., the difference between $\delta^{30} \mathrm{Si}$ in the solid and in the fluid).

Isotopic signatures of hydrothermal fluids are poorly constrained, but are most likely inherited from the igneous rocks through which the fluids percolate (cf., Robert and Chaussidon, 2006). They should therefore be around $-0.2 \%$ (i.e., mean value for crustal rocks; Fig. 4), which is in good agreement with available data for hydrothermal fluids (Fig. 4) (Ding et al., 1996; De La Rocha et al., 2000).

Because of the lack of experimental data on $\Delta_{\text {solid-fluid, }}$ we will adopt two different estimates: a value of $-2.2 \%$, based on the difference in $\delta^{30} \mathrm{Si}$ between our most negative chert sample $(-2.4 \%)$ and hydrothermal fluids $(-0.2 \%)$, and a value of $-3.0 \%$, approximating the difference between the most depleted hydrothermal deposits documented so far (Fig. 4) and a typical hydrothermal fluid. We consider these fractionation factors rather extreme values as they are more negative than those inferred for other inorganic processes such as silcretization, clay formation and silica adsorption onto Fe-oxides, which are on the order of $-1.5 \%$ (Basile-Doelsch et al., 2005; Georg et al., 2007b; Delstanche et al., 2009).

Furthermore, the degree of fractionation may change with the deposition temperature, in analogy to the oxygen isotope system (e.g., Matheney and Knauth, 1993), but this remains to be verified by experiments. Nonetheless, the cherts studied here mostly consist of microcrystalline quartz, which are typically transformation products of amorphous/opaline silica deposits such as sinters and diatom oozes (e.g., Williams and Crerar, 1985; Rodgers et al., 2004). As amorphous silica deposition is restricted to relatively low temperatures, we will ignore any temperature-dependent fractionation. Shifts in $\mathrm{Si}$ isotope composition associated with the transformation step are probably negligible and will not be taken into account (Douthitt, 1982).

Fig. $8 \mathrm{a}$ and $\mathrm{b}$ show the predicted $\delta^{30} \mathrm{Si}$ evolution of fluids and co-existing deposits during closed-system and open-system (modelled as Rayleigh) fractionation for $\Delta_{\text {solid-fluid }}=$
$-2.2 \%$ and $\Delta_{\text {solid-fluid }}=-3.0 \%$, respectively, based on the following equations:

Closed-system fractionation:

$$
\begin{aligned}
& \delta^{30} \mathrm{Si}_{\text {dissolved }}=\delta^{30} \mathrm{Si}_{\text {initial }}-\Delta *(1-f) \\
& \delta^{30} \mathrm{Si}_{\text {deposit }}=\delta^{30} \mathrm{Si}_{\text {initial }}+\Delta * f \\
& \text { Open-system fractionation: } \\
& \delta^{30} \mathrm{Si}_{\text {dissolved }}=\delta^{30} \mathrm{Si}_{\text {initial }}+\Delta * \ln (f) \\
& \delta^{30} \mathrm{Si}_{\text {deposit }}=\delta^{30} \mathrm{Si}_{\text {dissolved }}+\Delta
\end{aligned}
$$

where $f$ is the fraction of silica remaining in solution (Faure and Mensing, 2005).

5.2.2.2. Constraints imposed by the fractionation model. By virtue of its nature, closed-system fractionation cannot yield silica deposits with more positive $\delta^{30} \mathrm{Si}$ values than that of the starting solution. Open-system type fractionation could achieve this after a solution has lost enough precipitated silica. In order to generate chert with a $\delta^{30} \mathrm{Si}$ value as high as $+1.3 \%$ (our most positive $\delta^{30} \mathrm{Si}$ value found) from a hydrothermal fluid with an original $\delta^{30} \mathrm{Si}$ value of $-0.2 \%$, about $78-81 \%$ of its original silica content must have precipitated out $(f=0.22$ and $f=0.19$ for $\Delta_{\text {solid-fluid }}=-3.0 \%$ and $\Delta_{\text {solid-fluid }}=-2.2 \%$, respectively; see Fig. $8 \mathrm{a}$ and $\mathrm{b}$ ).

If it is assumed that silica precipitation from hydrothermal fluids depends on the difference between temperature and pressure conditions at the depth where fluids equilibrate with quartz and those at the surface (cf., Robert and Chaussidon, 2006), the silica concentration in the hydrothermal fluids can be calculated for a fixed seawater temperature (and thus silica concentration) and the estimates for $f$. We consider $0,25,55$ and $85^{\circ} \mathrm{C}$ as potential temperatures for Early Archaean oceans. The two highest temperatures represent the upper and lower limits of the 'hot' Archaean ocean model (Knauth and Lowe, 2003). The temperature of $25^{\circ} \mathrm{C}$ is an arbitrary intermediate value, while $0{ }^{\circ} \mathrm{C}$ is close to the lowest possible temperature of liquid water. Silica contents were calculated using the temperature-dependent solubility $(K)$ equation of Gunnarsson and Arnorsson (2000) for amorphous silica in pure water (cf., Robert and Chaussidon, 2006):

$$
\begin{aligned}
\log K_{\text {am.silica }}= & -8.476-485.24 \times T^{-1}-2.268 \times 10^{-6} \times T^{2} \\
& +3.068 \times \log T
\end{aligned}
$$

For each of these four scenarios, the silica concentration in hydrothermal fluid that would produce chert with $\delta^{30} \mathrm{Si}=+1.3 \%$ near a vent was calculated. Results are shown in Fig. 9, together with temperature and pressure-dependent solubility curves for quartz in seawater (Von Damm et al., 1991). The vertical grey box indicates the range of presentday high-temperature fluids from the East Pacific Rise (Von Damm et al., 1991). For the hottest seawater scenario $\left(85^{\circ} \mathrm{C}\right)$, the inferred silica concentration in hydrothermal fluid requires $P-T$ conditions in the reaction zone that would significantly exceed those in present-day hydrothermal sys- 
(a)
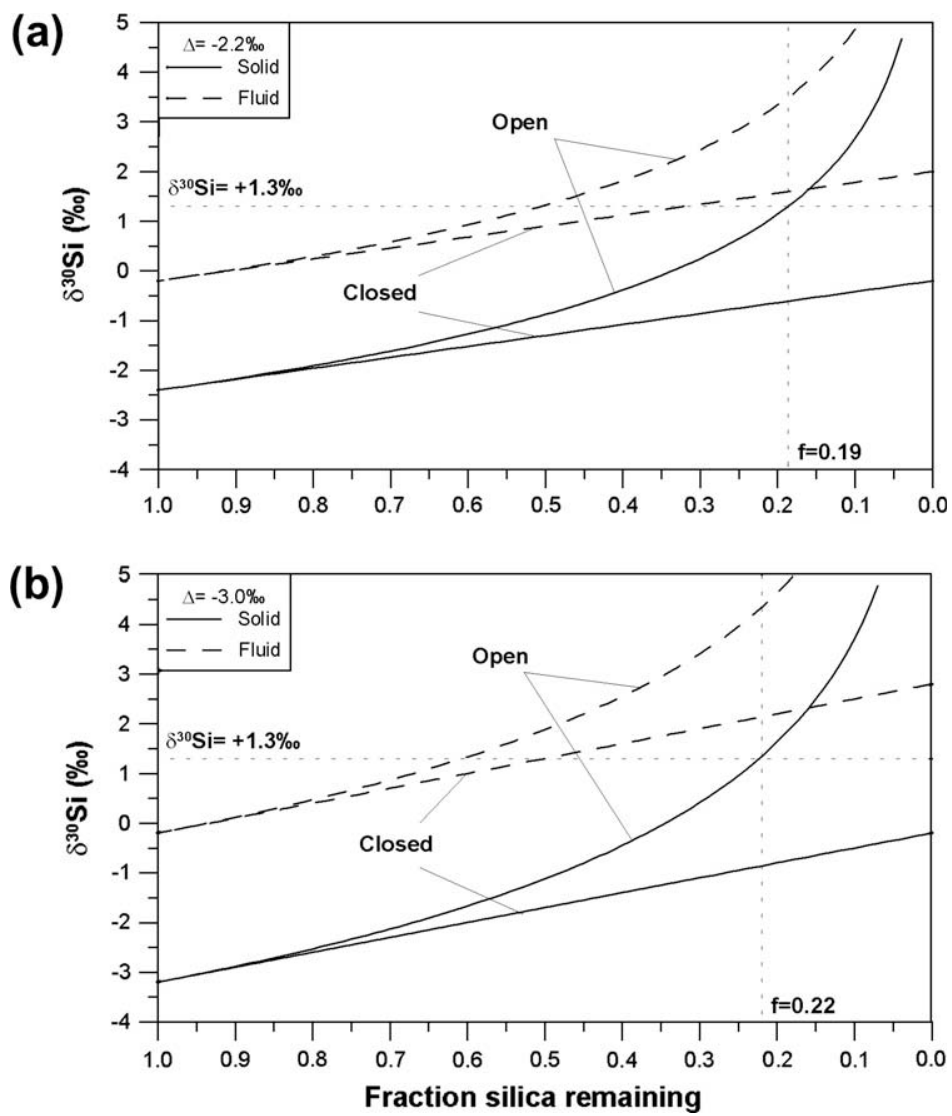

Fig. 8. Evolution of $\delta^{30} \mathrm{Si}$ in fluids and corresponding solids during progressive precipitation of silica, modelled as closed-system (linear) and Rayleigh-type (open-system) fractionation for (a) $\Delta_{\text {solid-fluid }}=-2.2$ and (b) $\Delta_{\text {solid-fluid }}=-3.0$. Dotted lines indicate $f$ at which solids attain $\delta^{30} \mathrm{Si}$ of $+1.3 \%$ o (see text for explanation).

tems ( $T<400{ }^{\circ} \mathrm{C}$ and pressure of a few hundred bars). The predicted hydrothermal conditions in the $55^{\circ} \mathrm{C}$ scenario largely overlap the $P-T$ range in modern settings.

\subsubsection{Conditions in Early Archaean hydrothermal systems.} However, it is conceivable that Early Archaean hydrothermal $P-T$ conditions - and therefore silica contents-were more moderate than today's. Less hydrostatic pressure due to lower ocean depths may have significantly reduced maximum equilibration temperatures of hydrothermal fluids in the subsurface (Kasting et al., 2006).

Increased melting at MORs in the Archaean, induced by more elevated mantle temperatures, generated thicker and thus more buoyant oceanic crust (Sleep and Windley, 1982), implying less isostatic difference between continents and oceans (Galer and Mezger, 1998), more water coverage onto the continents, and overall lower water depths. Supporting evidence for submersion of continents comes from the presence of zircon xenocrysts in subaqueous flood basalts (Arndt, 1999). Alternatively, continental landmass was still only a few percent of today and the oceans thus had more area to occupy (e.g., Reymer and Schubert, 1984; Collerson and Kamber, 1999).

Field evidence in the Pilbara and Barberton greenstone terranes confirms that sediments and volcanic extrusives were mainly deposited in shallow marine environments (e.g., Barley, 1993; Lowe, 1999). Sedimentary bed-forms in the Kitty's Gap and North Pole area are indicative of shallow water.

5.2.2.4. Preferred scenario for the formation of $C$-chert and seawater conditions. When adopting seawater temperatures of 0 and $25^{\circ} \mathrm{C}$, the calculations yield moderate hydrothermal silica contents that correspond to temperatures between 200 and $275^{\circ} \mathrm{C}$ in the reaction zone (Fig. 9), consistent with the alleged shallowness of hydrothermal convection. Hence, field evidence and observed Si isotope signatures offer a coherent scenario for the formation of Early Archaean C-chert near the interface of seawater and vent systems where fluids, which had equilibrated with crustal rocks under relatively low $P-T$, were issued. Nevertheless, temperatures up to about $55^{\circ} \mathrm{C}$ for Early Archaean seawater are conceivable from our modelling approach alone. Considering that our estimates for $\Delta_{\text {solid-fluid }}$ are maximum values and lower $\Delta_{\text {solid-fluid }}$ values would result in higher calculated silica contents in the hydrothermal fluids, hot-seawater scenarios with temperatures exceeding $55^{\circ} \mathrm{C}$ seem difficult to envisage.

Taking into account that (1) the most unequivocal involvement of seawater is seen in the S-cherts of Kitty's 


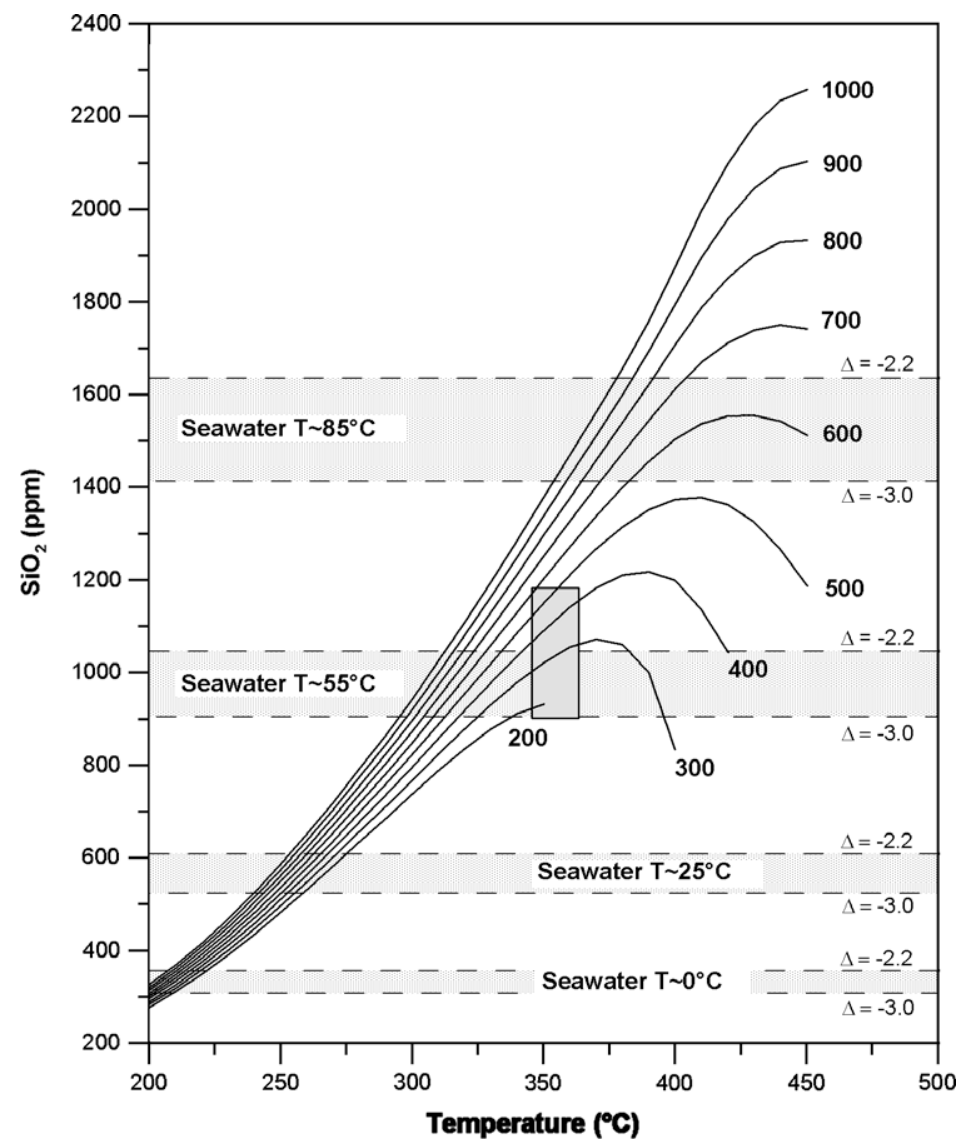

Fig. 9. Temperature-dependent isobaric (numbers indicate pressures in bars) solubility curves for quartz in seawater in the $200-450{ }^{\circ} \mathrm{C}$ temperature range (Von Damm et al., 1991; using temperature and pressure-dependent equation for seawater density from Bischoff and Rosenbauer, 1985). Vertical grey box indicates the region of present-day hydrothermal fluids at the East Pacific Rise (Von Damm et al., 1991). Horizontal grey bands indicate calculated silica contents of hydrothermal fluids for four scenarios where seawater is assumed to be saturated with amorphous silica at $0,25,55$ and $85^{\circ} \mathrm{C}$. Calculations were performed with $f$ values determined in Fig. 8 , based on $\Delta_{\text {solid-fluid }}$ values of -2.2 and -3.0 , which define the limits of the bands (see text for further explanation).

Gap, (2) the observed $\delta^{30} \mathrm{Si}$ values up to $+1.1 \%$ of these cherts point to ${ }^{30} \mathrm{Si}$-enrichment of seawater, and (3) this ${ }^{30} \mathrm{Si}$-enrichment cannot be due to silica-secreting organisms, nor to a prominent role of riverine input (if continental landmass was significantly smaller), we propose that globally averaged hydrothermal fluids lost enough silica to generate the ${ }^{30} \mathrm{Si}$-enrichment of Early Archaean seawater through a Rayleigh-distillation process (open-system fractionation). If correct, the above modelling approach argues against (very) high temperatures for ambient seawater. Furthermore, if the isotopic composition of seawater was largely governed by the input of evolved hydrothermal fluids, the distinction with seawater becomes rather arbitrary. Hence, it is difficult to evaluate whether the most positive $\delta^{30} \mathrm{Si}$ values in C-cherts reflect precipitation from evolved hydrothermal fluids, from "pure" seawater or from a mixture.

It should finally be noted that the overall $\delta^{30} \mathrm{Si}$ of seawater must reflect some balance between fractionated hydrothermal fluid inputs from on-axis ('high' temperature) and off-axis or ridge flank ('low' temperature) venting sites. Although off-axis hydrothermal fluids are generally less enriched in dissolved elements (including silica), their contri- bution might be important because off-axis seawater circulation encompasses a much greater area and volume than at ridge axes (Mottl and Wheat, 1994), even in the Archaean where ridges may have been twice as long as today (Howell, 1989). Hence, the estimated hydrothermal $P-T$ conditions (Fig. 9) represent an average for on- and off-axis inputs, which corroborates our conjecture that temperatures of $\sim 3.5 \mathrm{Ga}$ seawater were moderate. This line of reasoning implies that $\delta^{30} \mathrm{Si}$ of fractionated hydrothermal fluids at ridge axes might exceed that of seawater, especially if fluids would be strongly enriched in silica by diking events (Gillis, 1995), boiling, or phase separation. Even so, we maintain that the $\delta^{30} \mathrm{Si}$ value of $\sim+1.3 \%$ at North Pole is a good approximation of seawater chert, given the close agreement with values derived from the Kitty's Gap Chert. When adopting maximum fractionation factors of $-2.2 \%$ to $-3.0 \%$ for chemical precipitation of silica, and values of roughly $+1.3 \%$ representing seawater chert, Archaean seawater $\delta^{30} \mathrm{Si}$ would have been on the order of $+3 \%$ to $+4 \%$.

Such Archaean seawater $\delta^{30} \mathrm{Si}$ would significantly exceed present-day whole-ocean average values $(\mathrm{ca} .+1.1 \%$, De La 
Rocha et al., 2000) and compare to the most positive $\delta^{30} \mathrm{Si}$ of surface waters in the Southern Ocean (e.g., Varela et al., 2004). A tentative explanation might be that, although the global $\delta^{30} \mathrm{Si}$ signature of present-day ocean water is largely imparted by preferential uptake of ${ }^{28} \mathrm{Si}$ in diatoms, this effect is modulated by preferential release of ${ }^{28} \mathrm{Si}$ accompanying their partial re-dissolution (Demarest et al., 2009) and perhaps the initial stages of basalt weathering (Ziegler et al., 2005a). Such counter-effects will be minimal in a saturated silica-precipitating ocean (devoid of silica-secreting organisms), allowing the Early Archaean marine pool of dissolved silica to become more positive than today.

\subsection{Silicon isotope variations in three Early Archaean settings}

In the previous sections we explored two models that might account for the isotopic heterogeneity in cherts from the Pilbara craton. We showed that pure mixing of hydrothermal fluids and seawater is incapable of explaining the entire range in C-chert isotopic composition whereas the fractionation model on its own does not explain the $\mathrm{Al}_{2} \mathrm{O}_{3}$ vs. $\delta^{30} \mathrm{Si}$ trend in S-cherts. We therefore propose that both processes operated in the Early Archaean and together explain the observed isotopic heterogeneity.

\subsubsection{The Marble Bar Chert environment}

Marble Bar is a typical C-chert environment where Van Kranendonk (2006) identified three major phases of chert formation. The $\mathrm{Si}$ isotope composition of red chertsdeposited at the seafloor-water interface-is strongly variable and is consistent with dynamic mixing between hydrothermal fluids and seawater.

Shortly after deposition, the red chert was intruded by white chert, which is characterised by a more restricted range in $\delta^{30} \mathrm{Si}$ (Fig. 3). The intrusion of white chert might be associated with a phase of less vigorous hydrothermal activity. Hydrothermal fluids slowly percolated through the red-chert strata instead of being emanated directly into the oceans, which explains the more uniform $\delta^{30} \mathrm{Si}$ of white cherts. Furthermore, the low energy of hydrothermal activity probably enhanced conductive cooling of the fluids, resulting in losses of ${ }^{28} \mathrm{Si}$-enriched silica, which explain the only modest ${ }^{30} \mathrm{Si}$ depletion in white-chert samples (ca. $-0.3 \%$ ). This scenario would predict a gradual increase in $\delta^{30} \mathrm{Si}$ of white cherts from the base to the top of the Marble Bar unit, which is not apparent in Fig. 3, arguably due to the limited data set. An alternative explanation is that hydrothermal fluids mixed with seawater still present in pore/void spaces of the red chert. Because hydrothermal fluids were not in direct contact with the external seawater reservoir, this mixing was confined and resulted in uniform $\delta^{30} \mathrm{Si}$.

The white-red banded chert deposit was fragmented by the intrusion of black chert. The black-chert samples show a restricted range in $\delta^{30} \mathrm{Si}$ as well, which is consistent with their intrusive nature. Whether evolving hydrothermal fluids or mixing with seawater (see above) produced their slightly positive $\delta^{30} \mathrm{Si}$ values remains to be assessed. Presumably, both processes played a role. Possible differences in $\mathrm{Si}$ isotope fractionation between direct silica precipita- tion and adsorption onto Fe-hydroxides (Delstanche et al., 2009) may further add to inter-laminae heterogeneity.

The systematics in $\mathrm{Si}$ isotope signatures of the Marble Bar Chert types thus tend to fit into the genetic framework proposed by Van Kranendonk (2006). The adopted colourbased subdivision in our samples provides first-order evidence, but more rigorous sampling throughout the entire unit is required to further substantiate relationships between $\delta^{30} \mathrm{Si}$ signatures and the nature of successive chert forming phases.

\subsubsection{The Kitty's Gap Chert environment}

As explained in Section 5.2.1.1, $\mathrm{Si}$ isotope variability in Kitty's Gap S-cherts is largely the result of heterogeneous silicification of volcanogenic sediments by ${ }^{30} \mathrm{Si}$-enriched seawater. The extent of silicification is presumably a function of grain size, sorting and composition of precursor sediments.

Only two samples of almost pure silica show slightly depleted $\delta^{30} \mathrm{Si}$, which points to minor involvement of hydrothermal silica in the silicification process. This scarcity of isotopically depleted S-cherts suggests that ambient seawater was the main source of externally-derived silica for this type and that local hydrothermal activity was relatively weak at Kitty's Gap. For example, if hydrothermal fluids equilibrated with quartz in the reaction zone at temperatures below $\sim 150-175^{\circ} \mathrm{C}$, they remained undersaturated with respect to amorphous silica upon cooling (Fig. 7), so that no hydrothermal silica was deposited. In this case, the hydrothermal fluids remained largely unfractionated (i.e., $\delta^{30} \mathrm{Si}$ $\sim-0.2 \%$ ) until their emergence at the seafloor. The observed $\delta^{30} \mathrm{Si}$ values up to $+1.1 \%$ in the Kitty's Gap cherts suggests that such unfractionated fluids were insignificant in terms of controlling the Si isotope budget of Early Archaean seawater or were balanced by strongly fractionated signatures from other (high-T) fluids (Section 5.2.2.2).

The depositional environment of the Kitty's Gap Chert provides an alternative and perhaps more elegant explanation for the absence of a clear hydrothermal silica component. From a detailed sedimentological and petrographic study, De Vries (2004) proposed that sedimentation took place in a tidal-flat environment. Action of tidal currents could have promoted effective mixing between hydrothermal inputs and seawater, whereby the hydrothermal signature was obliterated (see also Section 5.3.3). At the same time, a tidally influenced setting, combined with high initial porosities of the sediments (Rouchon and Orberger, 2008) will imply very high water/sediment ratios, making it conceivable that the most silicified and ${ }^{30}$ Si-enriched S-cherts largely reflect silica from seawater and relatively little residual silica originating from the precursor.

The subordinate importance of 'hydrothermal silica' is further illustrated by the composition of the chert dikes that are mainly filled with silicified wall rock and surface sediments. Silicification of (fragmented) wall rock must have occurred via seawater percolating downwards along cracks and fractures that were associated with growth faulting. Given the presence of explosive (hydrothermal) breccias in the stratiform chert (Nijman et al., 1999b; De Vries, 2004) they presumably acted as conduits for hydrothermal fluids prior 
to being invaded by seawater. We thus propose that silicification largely succeeded hydrothermal activity here, which must have had a relatively low-temperature character so that chemical infilling of the conduits was largely inhibited.

\subsubsection{The North Pole Chert environment}

The North Pole Chert exhibits the most pronounced Si isotope variability of this study. The observed $\delta^{30} \mathrm{Si}$ as low as $-2.4 \%$ in stratiform chert sample VM180T indicates that, occasionally, mixing of pristine hydrothermal fluids with seawater was negligible. Over $2 \%$ more positive $\delta^{30} \mathrm{Si}$ of adjacent laminae in the same sample demonstrates the dynamic nature of hydrothermal activity. Such strong fluctuations reflect pulses of variably evolved hydrothermal fluids, interaction with seawater or a combination of both (cf., Nijman et al., 1999a; Van Kranendonk and Pirajno, 2004). The close association of white-red banded cherts (VM180T) with barite deposits (Nijman et al., 1999a) suggests that mixing between hydrothermal fluids and seawater played an important role in generating this isotopic heterogeneity (cf., Van Kranendonk, 2006).

The positive $\delta^{30} \mathrm{Si}$ of the silicified-sandstone sample (NPCU5) points to seawater as the dominant source of silica. It is of interest to note that the bulk $\delta^{30} \mathrm{Si}$ of this sample (NPCU5B) is considerably more positive $(\sim 0.5 \%)$ than that of the translucent cement of the groundmass (NP-CU5). The relative depletion of ${ }^{30} \mathrm{Si}$ in the silica cement probably signals a hydrothermal silica component. These observations strengthen our hypothesis that silicification of volcanogenic sediments occurs largely by seawater, whereas chemical deposition of silica requires hydrothermal fluids.

The contrast between the positive $\delta^{30} \mathrm{Si}$ of the sandstone sample and the ${ }^{30} \mathrm{Si}$-depletions in sample VM180T might be explained by differences in depositional environment. The sandstone sample belongs to lithofacies CU-S (Coarsening Upward Sandstone), interpreted to represent a tide-dominated deltaic environment (Nijman et al., 1999a). Hydrothermal venting in such a high-energy environment likely results in vigorous mixing with seawater, so that hydrothermal signals in the $\mathrm{Si}$ isotopes are readily diluted or overprinted. Sample VM180T, on the other hand, belongs to the ELC (Even Laminated Chert) facies (Nijman et al., 1999a), representing a low-energy subtidal basin-floor environment, possibly behind a coastal barrier, where mixing will be much less intense.

Interpretation of the $\mathrm{Si}$ isotope signatures of the North Pole dike cherts remains largely inconclusive, as they cannot be unambiguously assigned to one of the end-member types that we defined. If dikes are taken as S-cherts, $\delta^{30} \mathrm{Si}$ values down to $-1.1 \%$ would imply that silicification of precursor sediments occurred through interaction with hydrothermal fluids, in contrast to our previous findings. Alternatively, if they are C-cherts, their variable $\delta^{30} \mathrm{Si}$ values probably record different stages in the evolution of hydrothermal fluids. The resemblance of some of the REE $+Y$ patterns of dike chert samples with hydrothermal fluids lends support to a hydrothermal origin of the silica (cf., Nijman et al., 1999a; Ueno et al., 2004). Detailed sampling of dikes along vertical transects may expose isotopic trends that could solve this issue.

\subsection{Decoupling of REE+Y signatures and $\mathrm{Si}$ isotopes in chemical cherts}

The strong $\mathrm{La} / \mathrm{La}_{\mathrm{SN}}^{*}$ and $\mathrm{Y} / \mathrm{Ho}$ anomalies in many of the $\mathrm{C}$-chert samples, suggesting an important role of seawater in their formation, is consistent with the mixing model. Nonetheless, there is no obvious correlation between $\delta^{30} \mathrm{Si}$ and these anomalies (Fig. 5) nor with $\mathrm{Eu} / \mathrm{Eu}_{\mathrm{SN}}^{*}$ anomalies which would be expected if $\mathrm{Si}$ isotope and $\mathrm{REE}+\mathrm{Y}$ signatures are controlled by the same process. A translucent layer (G4) in sample VM180T is an extreme example in having the typical $\mathrm{La} / \mathrm{La}_{\mathrm{SN}}^{*}$ and $\mathrm{Y} / \mathrm{Ho}$ anomalies and low $\mathrm{Pr}_{\mathrm{SN}} / \mathrm{Yb}_{\mathrm{SN}}$ ratios of a seawater precipitate (cf., Bolhar et al., 2004, 2005; Friend et al., 2008) but being lowest in $\delta^{30} \mathrm{Si}$ of all. Its anomalous $\mathrm{Eu}$ abundance does, however, signal a hydrothermal component.

Hence, the $\mathrm{Si}$ isotopic compositions and $\mathrm{REE}+\mathrm{Y}$ signatures of cherts appear to be largely decoupled. As the $\mathrm{REE}+\mathrm{Y}$ inventory is most likely governed by Fe-(hydr)oxides and other particles rather than by silica, it is reasonable to suppose that the nature and amount of such impurities with incorporated and/or sorbed REEs and Y determine the extent of decoupling. In a mixing scenario it is conceivable that silica is largely hydrothermal in origin whereas metal-bearing particles precipitated after mixing with seawater. Alternatively, original REE $+\mathrm{Y}$ budgets in deposited cherts re-equilibrated with seawater by sorptive exchange on particles. Overprinting of hydrothermal signatures by seawater is also observed in modern hydrothermal vent deposits (German et al., 1990). Absence of seawater signatures in dikes of the North Pole Chert suggests that seawater may not have percolated downwards into the fractures here.

\subsection{Post-depositional alteration?}

A point of debate concerning the origin of Archaean cherts is the question of whether silicification occurred at or near the surface during deposition or through secondary alteration or metamorphic processes. Van den Boorn et al. (2007) pointed out that (1) marked (sub)cm-scale variations, (2) positive $\delta^{30} \mathrm{Si}$ up to $+1.1 \%$, and (3) field relations argue against pervasive metasomatic/metamorphic silicification or silcretization events (cf., André et al., 2006; Van Kranendonk, 2006).

However, it is conceivable that the small-scale $\mathrm{Si}$ isotope heterogeneity was generated by post-depositional carbonate replacement if primary deposits were composed of alternating carbonate-silica layers. Despite the absence of clear evidence for carbonate precursors in our samples, exploration of this mechanism is warranted, given the evidence for replaced carbonates in the North Pole locality (Buick and Dunlop, 1990). Carbonates are particularly prone to silicification (Knauth, 1994; Maliva et al., 2005), and (almost) complete conversion into microcrystalline quartz makes recognition of this precursor difficult. Also, seawater-inherited positive La, Eu and $\mathrm{Y}$ anomalies of Archaean carbonates (e.g., Van Kranendonk et al., 2003) may have (partially) survived alteration, and are thus not necessarily inconsistent with a replacement origin of alleged C-cherts that show these features. 
Intermittent or contemporaneous precipitation of silica and carbonate in modern environments has been reported from hydrothermal venting in thermal pools or coastal areas (Campbell et al., 2002; Canet et al., 2005). Silica deposition is here related to conductive cooling of near-neutral to slightly alkaline hydrothermal fluids, whereas carbonates precipitate upon evaporation or $\mathrm{CO}_{2}$ degassing (Campbell et al., 2002; Canet et al., 2005). Subsequent replacement of the carbonates could be induced by a more acidic hydrothermal event (cf., Van Kranendonk and Pirajno, 2004). Alternatively, carbonate layers represent periods of hydrothermal quiescence and precipitate from seawater, but are subsequently replaced by renewed hydrothermal activity.

Although the physical mechanism of carbonate replacement is different from primary silica deposition, major implications for the interpretation of $\mathrm{Si}$ isotope systematics in C-cherts are unlikely if replacement occurred shortly after deposition. In either case, mixing with seawater and/ or conductive cooling of hydrothermal fluids at shallow depths will govern fractionation of the $\mathrm{Si}$ isotopes. At North Pole there is evidence that silicification (and replacement of carbonate) occurred shortly after deposition and before substantial burial/deformation (Buick and Dunlop, 1990; Nijman et al., 1999a; Van Kranendonk, 2006). At Marble Bar the timing of any replacement would be more difficult to constrain, but the non-penetrative nature of chert veins also suggests that silicification occurred prior to substantial burial (Van Kranendonk, 2006). The overall early silicification of marine deposits in the Pilbara region thus substantiates the conclusion that $\mathrm{Si}$ isotope signatures of $\mathrm{C}$-cherts reflect near-surface mixing and cooling.

\subsection{Implications for $\mathrm{Si}$ isotope palaeothermometry}

Robert and Chaussidon (2006) reported a secular increase in $\delta^{30} \mathrm{Si}$ of cherts from 3.5 to $0.6 \mathrm{Ga}$, concomitant with a rise in the oxygen isotope composition of cherts over the same time span (Knauth and Lowe, 1978, 2003). These trends have been attributed to a decrease in ocean temperatures from $\sim 70{ }^{\circ} \mathrm{C}$ at $3.5 \mathrm{Ga}$ to present-day values by the end of the Proterozoic. Like our 'fractionation model' this 'palaeothermometer model' relies on the assumption that seawater $\delta^{30} \mathrm{Si}$ is predominantly controlled by the input of fractionated hydrothermal fluids. From the fit between $\delta^{18} \mathrm{O}$ and $\delta^{30} \mathrm{Si}$ in Precambrian cherts, the Robert and Chaussidon (2006) model yielded the temperature of hydrothermal fluids and the fractionation factors associated with precipitation of silica. Assuming that hydrothermal $P-T$ conditions remained constant throughout geologic history, they proposed that seawater temperature should have decreased in the 3.5-0.6 Ga interval in order to explain the secular increase in $\delta^{30} \mathrm{Si}$. Our model follows an inverse approach: for a set of adopted ocean temperatures and silica contents the corresponding conditions in hydrothermal venting systems are calculated. This allows assessing the conditions of the hydrothermal systems, including potential secular changes.

Irrespective of the validity for this alternative approach, our data for $3.5 \mathrm{Ga}$ cherts show a considerable range, including a maximum $\delta^{30} \mathrm{Si}$ value of ca. $+1.3 \%$, which chal- lenges the existence of a secular increase starting from ca. $0.0 \%$, as inferred by Robert and Chaussidon (2006). This primary isotopic variability poses the question of representativity when deducing an ocean temperature from an average $\delta^{30} \mathrm{Si}$ for a given age (Robert and Chaussidon, 2006). For example, adopting a $\delta^{30} \mathrm{Si}$ value of about $+1.3 \%$ would considerably lower the temperature estimate (average of $\sim 50{ }^{\circ} \mathrm{C}$ instead of $\sim 70{ }^{\circ} \mathrm{C}$ ) for this early time interval when following the approach of Robert and Chaussidon (2006).

Nonetheless, even if the trend can be confirmed in future work we surmise that it does not necessarily reflect a decrease in seawater temperature but could signal a secular increase in the temperature of hydrothermal fluids. As our fractionation model predicts, the $\delta^{30} \mathrm{Si}$ of seawater would rise when hydrothermal fluids become hotter and lose more ${ }^{28} \mathrm{Si}$ by silica precipitation before entering the ocean reservoir. Kasting et al. (2006) used similar reasoning to question evidence for hot Archaean oceans based on oxygen isotopes. Finally, any increase in seawater $\delta^{30} \mathrm{Si}$ could also be enhanced by an increase in the supply of ${ }^{30} \mathrm{Si}$-enriched riverine silica from growing continents (De La Rocha, 2006).

\section{CONCLUSIONS}

In this study we report a detailed set of mineralogical, geochemical and $\mathrm{Si}$ isotope data for three distinct, approximately $3.5 \mathrm{Ga}$ old chert deposits from the Pilbara greenstone belt (Western Australia). Silicon isotope variability in these cherts is large $\left(\delta^{30} \mathrm{Si}=-2.4 \%\right.$ to $+1.3 \%$ ond rules out secondary regional silicification events as principal cause of silica accumulation in Early Archaean rock sequences. Instead, we show that voluminous marine silica precipitation is due to elevated silica contents in the Archaean ocean and the input of Si-rich hydrothermal fluids. Our findings provide insightful information on the Early Archaean silica cycle, which can be summarised as follows.

- Archaean seawaters are enriched in ${ }^{30} \mathrm{Si}$ by the input of fractionated hydrothermal fluids and provide the main source of silica for extensive silicification of volcanogenic sediments, which results in the formation of S-cherts (silicified precursor cherts). The extent of silicification, possibly controlled by grain size of the original sediments, ultimately determines the $\mathrm{Si}$ isotope composition of S-cherts, which show a $\delta^{30} \mathrm{Si}$ range between $+0.1 \%$ and $+1.1 \%$.

- Mixing relationships indicate that injection of Si-rich hydrothermal fluids into Si-saturated seawater led to massive precipitation of silica as orthochemical deposits (C-cherts) in the vicinity of hydrothermal venting sites. Silicon isotope variability in C-cherts is extreme on hand-specimen as well as outcrop scale $\left(\delta^{30} \mathrm{Si}=-2.4 \%\right.$ o to $+0.6 \%$ ), and is inferred to reflect a combination of seawater mixing and fractionation by conductive cooling of hot fluids. Trace-element data confirm the chemical origin of these cherts, and equally point to seawater (positive La and Y anomalies) and hydrothermal fluids (positive Eu anomalies) as the dominant sources of silica. Absence of obvious correlations between REE $+\mathrm{Y}$ 
anomalies and $\delta^{30} \mathrm{Si}$ shows that these signatures are (partially) decoupled.

- The interpretation of trace element and Si isotope systematics in samples of dike chert is equivocal. Dike cherts are probably mixtures of silicified remains of wall rock, downward infiltrated seafloor sediments and chemically precipitated silica induced by hydrothermal activity.

- Deposition environments may control the extent to which hydrothermal $\mathrm{Si}$ isotope signatures are preserved in cherts. In shallow and tidally influenced settings hydrothermal signatures are largely lost due to more intensive mixing with seawater, whereas chemical cherts that are deposited in deeper parts of the ocean (i.e., below wave base) and/or under lower energy regimes retain strongly ${ }^{30} \mathrm{Si}$-depleted hydrothermal signatures.

- Using a simplified model that relates Si isotope fractionation in hydrothermal fluids to temperature differences with seawater (cf., Robert and Chaussidon, 2006), we demonstrate that chert deposits with $\delta^{30} \mathrm{Si}$ up to $+1.3 \%$ are difficult to reconcile with (very) hot Archaean seawater. Taking uncertainties into consideration, we propose $\sim 55^{\circ} \mathrm{C}$ as a rough upper limit for the temperature of the ocean $\sim 3.5 \mathrm{Ga}$ ago.

Although these findings were derived from $\mathrm{Si}$ isotope heterogeneity among cherts of the Pilbara greenstone terrane, similar processes have likely governed the origin of cherts in other Archaean cratons of similar age, such as the Barberton greenstone belt (South Africa). The demonstrated capability of Si isotopes to distinguish between different sources of silica will benefit future studies aiming to establish properties of Archaean fluids.

\section{ACKNOWLEDGMENTS}

We thank R. Smeets for dedicated support in the clean laboratory. B. van der Wagt, H. de Waard and T. Bouten are thanked for skilful assistance with (MC)-ICP-MS, ICP-AES and EMP analyses, respectively. Helpful comments of P. Van Cappellen and $\mathrm{H}$. Oonk are greatly appreciated. The manuscript benefited from critical and constructive reviews by M. Chaussidon, M. Bau and B. Georg, and from editorial comments of J. Alt. Fieldwork in the Pilbara region was financially supported by the Dr. Schürmannfonds Foundation (Grant 28/2005), and the Multi collector-inductively coupled plasma-mass spectrometer (MC-ICP-MS) facility was supported by a grant (175.107.404.01) from the Netherlands Organisation for Scientific Research (NWO-ALW).

\section{APPENDIX A. SUPPLEMENTARY DATA}

Supplementary data associated with this article can be found, in the online version, at doi:10.1016/j.gca.2009. 09.009 .

\section{REFERENCES}

Abraham K., Opfergelt S., Cardinal D., Hofmann A., Foley S. and André L. (2007) $\mathrm{Si}$ isotopes as a clue for understanding Eoarchaean silicifications. Geochim. Cosmochim. Acta Goldschmidt Conf. Abstr. 71, A3.
Alexander B. W., Bau M. and Andersson P. (2009) Neodymium isotopes in Archean seawater and implications for the marine $\mathrm{Nd}$ cycle in the Earth's early oceans. Earth Plan. Sci. Lett. 283, 144-155.

André L., Cardinal D., Alleman L. Y. and Moorbath S. (2006) Silicon isotopes in $3.8 \mathrm{Ga}$ West Greenland rocks as clues to the Eoarchaean supracrustal Si cycle. Earth Plan. Sci. Lett. 245, 162-173.

Arndt N. (1999) Why was flood volcanism on submerged continental platforms so common in the Precambrian. Precambrian Res. 97, 155-164.

Barley M. E. (1993) Volcanic, sedimentary and tectonostratigraphic environments of the $3.46 \mathrm{Ga}$ Warrawoona Megasequence: a review. Precambrian Res. 60, 47-67.

Basile-Doelsch I., Meunier J.-D. and Parron C. (2005) Another continental pool in the terrestrial silicon cycle. Nature 433, 399402.

Bau M. (1996) Controls on the fractionation of isovalent trace elements in magmatic and aqueous systems: evidence from $\mathrm{Y} /$ $\mathrm{Ho}, \mathrm{Zr} / \mathrm{Hf}$ and lanthanide tetrad effect. Contrib. Mineral. Petrol. 123, 323-333.

Bau M. and Dulski P. (1996) Distribution of yttrium and rare earth elements in the Penge and Kuruman iron formations, Transvaal Supergroup, South Africa. Precambrian Res. 79, 37-55.

Bischoff J. L. and Rosenbauer R. J. (1985) An empirical equation of state for hydrothermal seawater (3.2 percent $\mathrm{NaCl}$ ). Am. J. Sci. 285, 725-763.

Bolhar R., Kamber B. S., Moorbath S., Fedo C. M. and Whitehouse M. J. (2004) Characterization of early Archaean chemical sediments by trace element signatures. Earth Plan. Sci. Lett. 222, 43-60.

Bolhar R., Van Kranendonk M. J. and Kamber B. S. (2005) A trace element study of siderite-jasper banded iron formation in the $3.45 \mathrm{Ga}$ Warrawoona Group, Pilbara Craton-formation from hydrothermal fluids and shallow water. Precambrian Res. 137, 93-114.

Buick R. and Dunlop J. S. R. (1990) Evaporitic sediments of early Archean age from the Warrawoona Group, North Pole, Western Australia. Sedimentology 37, 247-277.

Buick R., Thornett J. R., McNaughton N. J., Smith J. B., Barley M. E. and Savage M. (1995) Record of emergent continental crust 3.5 billion years ago in the Pilbara craton of Australia. Nature 375, 574-577.

Campbell K. A., Rodgers K. A., Botheridge J. M. A. and Browne P. R. L. (2002) An unusual modern silica-carbonate sinter from Pavlova spring, Ngatamariki, New Zealand. Sedimentology 49, 835854.

Canet C., Prol-Ledesma R. M., Torres-Alvarado I., Gilg H. A., Villanueva R. E. and Lozano-Santa Cruz R. (2005) Silicacarbonate stromatolites related to coastal hydrothermal venting in Bahía Concepción, Baja California Sur, Mexico. Sediment. Geol. 174, 97-113.

Cardinal D., Alleman L. Y., Dehairs F., Savoye N., Trull T. W. and André L. (2005) Relevance of silicon isotopes to Si-nutrient utilization and Si-source assessment in Antarctic waters. Global Biogeochem. Cycles 19. doi:10.1029/2004GB002364.

Collerson K. D. and Kamber B. S. (1999) Evolution of the continents and the atmosphere inferred from Th-U-Nb systematics of the depleted mantle. Science 283, 1519-1522.

De La Rocha C. L., Brzezinski M. A. and DeNiro M. J. (1997) Fractionation of silicon isotopes by marine diatoms during biogenic silica formation. Geochim. Cosmochim. Acta 61, 50515056.

De La Rocha C. L., Brzezinski M. A. and DeNiro M. J. (2000) A first look at the distribution of the stable isotopes of silicon in natural waters. Geochim. Cosmochim. Acta 64, 2467-2477. 
De La Rocha C. L. (2003) Silicon isotope fractionation by marine sponges and the reconstruction of the silicon isotope composition of ancient deep water. Geology 31, 423-426.

De La Rocha C. L. (2006) Palaeoceanography in hot water. Nature 443, 920-921.

Delstanche S., Opfergelt S., Cardinal D., Elsass F., Andre L. and Delvaux B. (2009) Silicon isotopic fractionation during adsorption of aqueous monosilicic acid onto iron oxide. Geochim. Cosmochim. Acta 73, 923-934.

Demarest M. S., Brzezinski M. A. and Beucher C. P. (2009) Fractionation of silicon isotopes during biogenic silica dissolution. Geochim. Cosmochim. Acta 73, 5572-5583.

De Vries S. T. (2004) Early Archaean sedimentary basins: depositional environment and hydrothermal systems. Geol. Ultraiectina 244, 1-160.

De Vries S. T., Nijman W., Wijbrans J. R. and Nelson D. R. (2006) Stratigraphic continuity and early deformation of the central part of the Coppin Gap Greenstone Belt, Pilbara, Western Australia. Precambrian Res. 147, 1-27.

DiMarco M. J. and Lowe D. R. (1989a) Petrography and provenance of silicified early archean volcaniclastic sandstones, eastern Pilbara Block, Western Australia. Sedimentology 36, 821-836.

DiMarco M. J. and Lowe D. R. (1989b) Shallow water volcaniclastic deposition in the Early Archean Panorama formation, Warrawoona Group, eastern Pilbara Block, Western Australia. Sediment. Geol. 64, 43-63.

Ding T., Jiang S., Wang D., Li Y., Li J., Song H. and Lao X. (1996) Silicon Isotope Geochemistry. Geological Publishing House.

Ding T., Wan D., Wang C. and Zhang F. (2004) Silicon isotope compositions of dissolved silicon and suspended matter in the Yangtze river, China. Geochim. Cosmochim. Acta 68, 205-216.

Douthitt C. B. (1982) The geochemistry of the stable isotopes of silicon. Geochim. Cosmochim. Acta 46, 1449-1458.

Eggins S. M., Woodhead J. D., Kinsley L. P. J., Mortimer G. E., Sylvester P., McCulloch M. T., Hergt J. M. and Handler M. R. (1997) A simple method for the precise determination of $>40$ trace elements in geological samples by ICPMS using enriched isotope internal standardisation. Chem. Geol. 134, 311-326.

Faure G. and Mensing T. M. (2005) Isotopes: Principles and Applications. John Wiley \& Sons, New Jersey.

Foriel J., Philippot P., Rey P., Somogyi A., Banks D. and Menez B. (2004) Biological control of $\mathrm{Cl} / \mathrm{Br}$ and low sulfate concentration in a 3.5-Gyr-old seawater from North Pole, Western Australia. Earth Plan. Sci. Lett. 228, 451-463.

Fournier R. O. (1985). Silica minerals as indicators of conditions during gold deposition. In: Geological characteristics of sediment- and volcanic-hosted disseminated gold deposits search for an occurrence model (ed. E. W. Tooker), pp. 15-26.

Friend C. R. L., Nutman A. P., Bennett V. C. and Norman M. D. (2008) Seawater-like trace element signatures (REE + Y) of Eoarchaean chemical sedimentary rocks from southern West Greenland, and their corruption during high-grade metamorphism. Contrib. Mineral. Petrol. 155, 229-246.

Galer S. J. G. and Mezger K. (1998) Metamorphism, denudation and sea level in the Archean and cooling of the Earth. Precambrian Res. 92, 389-412.

Georg R. B., Reynolds B. C., Frank M. and Halliday A. N. (2006) Mechanisms controlling the silicon isotopic composition of river waters. Earth Plan. Sci. Lett. 249, 290-306.

Georg R. B., Halliday A. N., Schauble E. A. and Reynolds B. C. (2007a) Silicon in the Earth's core. Nature 447, 1102-1106.

Georg R. B., Reynolds B. C., West A. J., Burton K. W. and Halliday A. N. (2007b) Silicon isotope variations accompanying basalt weathering Iceland. Earth Plan. Sci. Lett. 261, 476-490.
German C. R., Klinkhammer G. P., Edmond J. M., Mitra A. and Elderfield H. (1990) Hydrothermal scavenging of rare earth elements in the ocean. Nature 345, 516-518.

Gillis K. M. (1995) Controls on hydrothermal alteration in a section of fast-spreading oceanic crust. Earth Plan. Sci. Lett. 34, 473-489.

Green M. G., Sylvester P. J. and Buick R. C. (2000) Growth and recycling of early Archean continental crust: geochemical evidence from the Coonterunah and Warrawoona Groups, Pilbara, Craton, Australia. Tectonophysics 322, 69-88.

Gunnarsson I. and Arnorsson S. (2000) Amorphous silica solubility and the thermodynamic properties of $\mathrm{H}_{4} \mathrm{SiO}_{4}$ in the range of

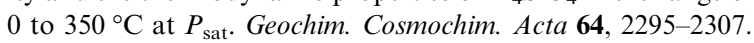

Hayashi T., Tanimizu M. and Tanaka T. (2004) Origin of negative $\mathrm{Ce}$ anomalies in Barberton sedimentary rocks, deduced from $\mathrm{La}-\mathrm{Ce}$ and $\mathrm{Sm}-\mathrm{Nd}$ isotope systematics. Precambrian Res. 135, 345-357.

Howell D. G. (1989) How the growth and freeboard of continents may relate to geometric and kinematic parameters of mid-ocean spreading ridges. Tectonophysics 161, 343-349.

Janecky D. R. and Seyfried W. E. (1984) Formation of massive sulfide deposits on ridge crests: incremental reaction models for mixing between hydrothermal solutions and seawater. Geochim. Cosmochim. Acta 48, 2723-2738.

Kamber B. S., Greig A. and Collerson K. D. (2005) A new estimate for the composition of weathered young upper continental crust from alluvial sediments, Queensland, Australia. Geochim. Cosmochim. Acta 69, 1041-1058.

Kasting J. F., Tazewell Howard M., Wallman K., Veizer J., Shields G. and Jaffrés J. (2006) Paleoclimates, ocean depth, and the oxygen isotopic composition of seawater. Earth Plan. Sci. Lett. 252, 82-93.

Kato Y. and Nakamura K. (2003) Origin and global tectonic significance of early Archean cherts from the Marble Bar greenstone belt, Pilbara Craton, Western Australia. Precambrian Res. 125, 191-243.

Kitajima K., Maruyama S., Utsunomiya S. and Liou J. G. (2001) Seafloor hydrothermal alteration at an Archean mid-oceanic ridge. J. Metamorph. Geol. 19, 581-597.

Knauth L. P. and Lowe D. R. (1978) Oxygen isotope geochemistry of cherts from the Onverwacht Group (3.4.billion years), Transvaal, South Africa, with implications for secular variations in the isotopic composition of cherts. Earth Plan. Sci. Lett. 41, 209-222.

Knauth L. P. (1994) Petrogenesis of chert. In Silica: Physical Behaviour, Geochemistry and Materials Applications. Reviews in Mineralogy \& Geochemistry, vol. 29 (eds. P. J. Heany, C. T. Prewitt and G. V. Gibbs). Mineralogical Society of America, pp. 233-256.

Knauth L. P. and Lowe D. R. (2003) High Archean climatic temperature inferred from oxygen isotope geochemistry of cherts in the $3.5 \mathrm{Ga}$ Swaziland Supergroup, South Africa. Geol. Soc. Am. Bull. 115, 566-580.

Kojima S., Hanamuro T., Hayashi K., Haruna M. and Ohmoto H. (1998) Sulphide minerals in Early Archean chemical sedimentary rocks of the eastern Pilbara district, Western Australia. Mineral. Petrol. 64, 219-235.

Krapez B. and Eisenlohr B. (1998) Tectonic settings of Archean (3325-2775) crustal-supracrustal belts in the West Pilbara Block. Precambrian Res. 88, 173-205.

Krauskopf K. B. (1956) Dissolution and precipitation of silica at low temperatures. Geochim. Cosmochim. Acta 10, 1-26.

Kristall B., Kelley D. S., Hannington M. D. and Delaney R. (2006) Growth history of a diffusely venting sulfide structure from the Juan de Fuca Ridge: a petrological and geochemical study. Geochem. Geophys. Geosyst. 7. doi:10.1029/2005GC001166. 
Lowe D. R. (1999) Petrology and sedimentology of cherts and related silicified sedimentary rocks in the Swaziland Supergroup. In Geological evolution of the Barberton Greenstone Belt, South Africa (ed. D. R. Lowe and G. R. Byerly). Geological Society of America Special Paper, vol. 329, pp. 83-114.

Lowe D. R. and Fisher Worrell G. (1999) Sedimentology, mineralogy, and implications of silicified evaporites in the Kromberg Formation, Barbeton Greenstone Belt, South Africa. In Geological evolution of the Barberton Greenstone Belt, South Africa (ed. D. R. Lowe and G. R. Byerly). Geological Society of America Special Paper, vol. 329, pp. $167-188$.

Maliva R. G., Knoll A. H. and Simonson B. M. (2005) Secular changes in the Precambrian silica cycle: insights from chert petrology. Geol. Soc. Am. Bull. 117, 835-845.

Matheney R. K. and Knauth L. P. (1993) New isotopic temperature estimates for early silica diagenesis in bedded chert. Geology 21, 519-522.

Mottl M. J. and Wheat C. G. (1994) Hydrothermal circulation though mid-ocean ridge flanks: fluxes of heat and magnesium. Geochim. Cosmochim. Acta 58, 2225-2237.

Nijman W., De Bruijne C. H. and Valkering M. E. (1999a) Growth fault control of early Archean cherts, barite mounds and chertbarite veins, North Pole Dome, Eastern Pilbara, Western Australia (second corrected edition). Precambrian Res. 95, 247274.

Nijman W., Willigers B. J. A. and Krikke A. (1999b) Tensile and compressive growth structures: relationships between sedimentation, deformation and granite intrusion in the Archaean Coppin Gap greenstone belt, Eastern Pilbara, Western Australia (second corrected edition). Precambrian Res. 95, 277-302.

Nishizawa M., Sano Y., Ueno Y. and Maruyama S. (2007) Speciation and isotope ratios of nitrogen in fluid inclusions from seafloor hydrothermal deposits at $3.5 \mathrm{Ga}$. Earth Plan. Sci. Lett. 254, 332-344.

Oliver N. H. S. and Cawood P. A. (2001) Early tectonic dewatering and brecciation on the overturned sequence at Marble Bar, Pilbara Craton, Western Australia: dome-related or not? Precambrian Res. 105, 1-15.

Orberger B., Rouchon V., Westall F., De Vries S. T., Pinti D. L., Wagner C., Wirth R. and Hashizume K. (2006) Microfacies and origin of some Archean cherts (Pilbara, Australia). Geol. Soc. Am. Special Papers 205, 133-156.

Reymer A. and Schubert G. (1984) Phanerozoic addition rates to the continental crust and crustal growth. Tectonics 3, 63-77.

Reynolds B. C., Frank M. and Halliday A. N. (2006) Silicon isotope fractionation during nutrient utilization in the North Pacific. Earth Plan. Sci. Lett. 244, 431-443.

Reynolds B. C., Aggarwal J., André L., Baxter D., Beucher C., Brzezinski M. A., Engström E., Georg R. B., Land M., Leng M. J., Opfergelt S., Rodushkin I., Sloane H. J., Van den Boorn S. H. J. M., Vroon P. Z. and Cardinal D. (2007) An interlaboratory comparison of $\mathrm{Si}$ isotope reference materials. $J$. Anal. At. Spectrom. 22, 561-568.

Rimstidt J. D. and Cole D. R. (1983) Geothermal mineralization I: the mechanism of formation of the Beowawe Nevada siliceous sinter deposit. Am. J. Sci. 283, 861-875.

Robert F. and Chaussidon M. (2006) A palaeotemperature curve for the Precambrian oceans based on silicon isotopes in cherts. Nature 443, 969-972.

Rodgers K. A., Browne P. R. L., Buddle T. F., Cook K. L., Greatrex R. A., Hampton W. A., Herdianita N. R., Holland G. R., Lynne B. Y., Martin R., Newton Z., Pastars D., Sannazaro K. L. and Teece C. I. A. (2004) Silica phases in sinters and residues from geothermal fields of New Zealand. Earth Sci. Rev. 66, 1-61.
Rouchon V. and Orberger B. (2008) Origin and mechanisms of KSi metasomatism of ca.3.4-3.3 Ga volcaniclastic deposits and implications for Archean seawater evolution: Examples from cherts of Kitty's Gap (Pilbara Craton, Australia) and Msauli (Barberton Greenstone Belt, South Africa). Precambrian Res. 165, 169-189.

Schopf J. W., Kudryavtsev A. B., Agresti D. G., Wdowiak T. J. and Czaja A. D. (2002) Laser-Raman imagery of Earth's earliest fossils. Nature 416, 73-76.

Seyfried W. E. (1987) Experimental and theoretical constraints on hydrothermal alteration processes at mid-ocean ridges. Ann. Rev. Earth Plan. Sci. 15, 317-335.

Siever R. (1992) The silica cycle in the Precambrian. Geochim. Cosmochim. Acta 56, 3265-3272.

Sleep N. H. and Windley B. F. (1982) Archaean plate tectonics: constraints and inferences. J. Geol. 90, 363-379.

Stüben D., Eddine Taibi N., McCuthry G. M., Scholten J., Stoffers P. and Zhang D. (1994) Growth history of a hydrothermal silica chimney from the Mariana backarc spreading centre (southwest Pacific, $\left.18^{\circ} 13^{\prime} \mathrm{N}\right)$. Chem. Geol. 113, 273-296.

Sugitani K. (1992) Geochemical characteristics of Archean cherts and other sedimentary rocks in the Pilbara Block, Western Australia: evidence for Archean seawater enriched in hydrothermally-derived iron and silica. Precambrian Res. 57, 21-47.

Sugitani K., Horiuchi Y., Adachi M. and Sugisaki R. (1996) Anomalously low $\mathrm{Al}_{2} \mathrm{O}_{3} / \mathrm{TiO}_{2}$ values of archean cherts from the Pilbara block, Western Australia_-possible evidence for extensive chemical weathering on the early earth. Precambrian Res. 80, 49-76.

Sugitani K., Yamamoto K., Adachi M., Kawabe I. and Sugisaki R. (1998) Archean cherts derived from chemical, biogenic and clastic sedimentation in a shallow restricted basin: examples from the Gorge Creek Group in the Pilbara block. Sedimentology 45, 1045-1062.

Ueno Y., Yoshioka H., Maruyama S. and Isozaki Y. (2004) Carbon isotopes and petrography of kerogens in $3.5 \mathrm{Ga}$ hydrothermal silica dikes in the North Pole area, Western Australia. Geochim. Cosmochim. Acta 68, 573-589.

Ueno Y., Yamada K., Yoshida N., Maruyama S. and Isozaki Y. (2006) Evidence from fluid inclusions for microbial methanogenesis in the early Archean era. Nature 440, 516-519.

Van den Boorn S. H. J. M., Vroon P. Z., van Belle C. C., van der Wagt B., Schwieters J. and van Bergen M. J. (2006) Determination of silicon isotope ratios in silicate materials by highresolution MC-ICP-MS using a sodium hydroxide sample digestion method. J. Anal. At. Spectrom. 21, 734-742.

Van den Boorn S. H. J. M., Van Bergen M. J., Nijman W. and Vroon P. Z. (2007) Dual role of seawater and hydrothermal fluids in Early Archean chert formation: evidence from silicon isotopes. Geology 35, 939-942.

Van der Meer J. (2002) Magmatic evolution in the Archean: new vidence rom the $\sim 3.5 \mathrm{Ga}$ old Duffer Formation, East Pilbara craton, Western Australia. Unpublished M.Sc. thesis, Utrecht University.

Van Kranendonk M. J., Hickman A. H., Smithies R. H., Nelson D. R. and Pike G. (2002) Geology and tectonic evolution of the Archean North Pilbara terrain, Pilbara craton, Western Australia. Econ. Geol. 97, 695-732.

Van Kranendonk M. J., Webb G. E. and Kamber B. S. (2003) Geological and trace element evidence for a marine sedimentary environment of deposition and biogenicity of $3.45 \mathrm{Ga}$ stromatolitic carbonates in the Pilbara craton, and support for a reducing Archaean ocean. Geobiology 1, 91-108.

Van Kranendonk M. J. and Pirajno F. (2004) Geochemistry of metabasalts and hydrothermal alteration zones associated with c. $3.45 \mathrm{Ga}$ chert and barite deposits: implication for the 
geological setting of the Warrawoona Group, Pilbara Craton, Australia. Geochem. Expl. Env. Anal. 4, 253-278.

Van Kranendonk M. J. (2006) Volcanic degassing, hydrothermal circulation and the flourishing of early life on Earth: a review of the evidence from c. 3490-3240 Ma rocks of the Pilbara Supergroup, Pilbara Craton, Western Australia. Earth Sci. Rev. 74, 197-240.

Van Kranendonk M. J., Smithies R. H., Hickman A. H. and Champion D. C. (2007) Review: secular tectonic evolution of Archean continental crust: interplay between horizontal and vertical processes in the formation of the Pilbara Craton, Australia. Terra Nova 19, 1-38.

Varela D. E., Pride C. J. and Brzezinski M. A. (2004) Biological fractionation of silicon isotopes in Southern Ocean surface waters. Global Biogeochem. Cycles 18. doi:10.1029/2003GB002140.

Von Damm K. L., Edmond J. M., Grant B. G. and Measures C. I. (1985) Chemistry of submarine hydrothermal solutions at $21^{\circ} \mathrm{N}$, East Pacific Rise. Geochim. Cosmochim. Acta 49, 2197-2220.

Von Damm K. L., Bischoff J. L. and Rosenbauer R. J. (1991) Quartz solubility in hydrothermal seawater: an experimental study and equation describing quartz solubility for up to $0.5 \mathrm{M}$ $\mathrm{NaCl}$ solutions. Am. J. Sci. 291, 977-1007.

Vroon P. Z., Van den Boorn S. H. J. M., Van der Wagt B. and Van Bergen M. J. (2007) In-situ silicon isotope analysis of Archean cherts by laser ablation MC-ICPMS. Eos Trans. AGU Fall Meet. Suppl. Abstr. 88, V51B-0565.

Walter M. R., Buick R. and Dunlop J. S. R. (1980) Stromatolites 3.400-3.500 Myr old from the North Pole area, western Australia. Nature 284, 443-445.

Westall F., De Vries S. T., Nijman W., Rouchon V., Orberger B., Pearson V., Watson J., Verchovsky A., Wright I., Rouzaud J.N., Marchesini D. and Severine A. (2006) The $3.466 \mathrm{Ga}$ "Kitty's Gap Chert," an Early Archean microbial ecosystem. Geol. Soc. Am. Special Papers 405.

Williams L. A. and Crerar D. A. (1985) Silica diagenesis, II. General mechanisms. J. Sediment. Petrol. 55, 312-321.

Ziegler K., Chadwick O. A., Brzezinski M. A. and Kelly E. F. (2005a) Natural variations of $\delta^{30} \mathrm{Si}$ ratios during progressive basalt weathering, Hawaiian Islands. Geochim. Cosmochim. Acta 19, 4597-4610.

Ziegler K., Chadwick O. A., White A. F. and Brzezinski M. A. (2005b) $\Delta^{30} \mathrm{Si}$ systematics in a granitic saprolite, Puerto Rico. Geology 33, 817-820. 